\title{
PERFIL DE ÉXITO DE ESTUDIANTES DE NUEVO INGRESO EN LA CARRERA DE TURISMO
}

\author{
Silvia Caballero-Soria* \\ José Antonio Camúñez Ruiz** \\ José Luis Jiménez-Caballero ${ }^{* * *}$ \\ Universidad de Sevilla
}

\section{RESUMEN}

El objetivo de este trabajo es identificar los factores que pueden contribuir a mejorar la asignación de la demanda de estudiantes de primer ingreso en una Facultad de Turismo. Para una muestra de 572 alumnos/as de la Universidad de Sevilla se pretende modelizar, usando una regresión cuantil complementaria a la estimación de mínimos cuadrados ordinarios clásica, los resultados académicos en las diferentes disciplinas impartidas en el primer curso para examinar el efecto de la nota de acceso, el sexo y el orden de preferencia con el que los estudiantes accedieron al Grado en Turismo. Los resultados del estudio sugieren que el orden de preferencia y la nota de acceso son variables significativas. La regresión cuantil permite identificar el peso de esas variables en los diferentes tramos del recorrido del resultado académico. Sobre la base de estos resultados se presentan implicaciones prácticas y sociales, y futuras líneas de investigación.

Palabras clave: Eficiencia; desempeño académico; gestión de recursos públicos; sexo; nota de acceso; orden de preference; regresion cuantil; regression por mínimos cuadrados ordinarios.

Classification JEL: M20, M21

Recibido: 5 de octubre de 2017

Devuelto para su revisión: 18 de diciembre de 2017

Aceptado: 2 de febrero de 2018

* Departamento de Economía Financiera y Dirección de Operaciones. Facultad de Turismo y Finanzas. Universidad de Sevilla. Avda. San Francisco Javier, s/n. 41018 Sevilla (España). E-mail:silviacs@ono.com.

** Departamento de Economía Aplicada I. Facultad de Ciencias Económicas y Empresariales. Universidad de Sevilla. Avda Ramón y Cajal, 1,41018 Sevilla. E-mail: camunez@us.es

*** Departamento de Economía Financiera y Dirección de Operaciones. Facultad de Turismo y Finanzas. Universidad de Sevilla. Avda. San Francisco Javier, s/n. 41018 Sevilla (España).E-mail: jjimenez@us.es. 


\title{
Success profile of incoming first year undergraduate students of tourism
}

\begin{abstract}
The purpose of this study is to identify the factors that can contribute to improving the allocation of new intake student demand at a university School of Tourism. For a sample of 572 students at the University of Seville, we model academic results in the different disciplines taught during the first year using quantile regression to complement estimation by classic ordinary least squares, in order to examine the effects of the admission grade, gender and the position of the Degree in Tourism on students' order of preference for them to get into university. Study results suggest that the order of preference and the admission grade are significant variables. Quantile regression allows the weights of these variables to be identified at different stages of the academic outcome. Practical and social implications are presented on the basis of these results along with future lines of research.
\end{abstract}

Keywords: Efficiency; Academic Performance; Public Administration of Resources; Gender; University Entrance Exam Grade; Order of Preference; Quantile Regression; Ordinary Least Squares (OLS) Regression.

\section{INTRODUCCIÓN}

La contribución del turismo al crecimiento del PIB y al empleo constituye a esta industria como una de las de mayor alcance para lograr el bienestar económico y social. Según la Organización Mundial del Turismo esta industria representa en 2016 el 10\% del PIB mundial y genera 1 de cada 11 empleos. La alta tasa de desempleo estructural en países desarrollados como España (tercer destino mundial y segundo país en volumen de ingresos turísticos en 2016) hace de la formación un elemento indispensable para lograr desarrollar profesionales que puedan adaptarse y encajar a lo que demanda hoy día el sector del turismo. La formación universitaria, como formación para el empleo cualificado, debe lograr que sus egresados alcancen un nivel de calidad y capacitación máximo. Para ello, es necesario, en primer lugar, que los estudios turísticos se adapten permanentemente a las demandas profesionales del sector; y, en segundo lugar, que el perfil de los estudiantes que acceden a una titulación universitaria en turismo sea el requerido para lograr un alto nivel de desempeño y resultados. Se trata, así, de analizar el perfil y condicionantes que contribuyen a formar los egresados más capacitados para lograr el éxito profesional.

Al mismo tiempo, las autoridades universitarias muestran un interés particular por los resultados académicos de sus estudiantes, cuyo estudio y análisis constituyen herramientas sólidas para construir indicadores que orienten la toma de decisiones en educación superior (Garbanzo, 2007).

Vamos a plantear, por tanto, como problema de investigación una propuesta de identificación de los factores que pueden contribuir a mejorar la asignación de la demanda de estudiantes de primer ingreso en una Facultad de Turismo. Se intenta, así, que ello pueda contribuir a un aumento del rendimiento académico, a una mejora de las capacidades alcanzadas por los egresados, $\mathrm{y}$, en consecuencia, a un incremento en la eficiencia 
de la gestión de recursos. No es deseable que ciertos estudiantes terminen en carreras no elegidas en primeras opciones: ello puede afectar su rendimiento académico y contribuir al abandono.

Por tanto, el propósito del estudio es examinar el efecto que tienen la nota de acceso, el sexo y el orden de preferencia con el que los estudiantes accedieron al Grado en Turismo sobre los resultados académicos de primer curso. Para cumplir este objetivo, en primer lugar, se aborda el marco teórico del estudio, y la metodología empleada: muestra utilizada y procedimiento estadístico utilizado. Se continúa con la exposición de los resultados obtenidos, terminando con las conclusiones del estudio.

\section{REVISIÓN DE LA LITERATURA}

Para Garbanzo (2007, p. 47): “Conocer los diferentes factores que inciden en el rendimiento académico en el campo de la educación superior de una manera más integral, permite obtener resultados tanto cualitativos como cuantitativos para propiciar un enfoque más completo en la toma decisiones para mejorar los niveles de pertinencia, equidad y calidad educativa". Hay bastantes estudios que han tratado de descubrir los factores que determinan la eficiencia académica en el mundo de la educación y las percepciones de los estudiantes hacia las titulaciones universitarias. La publicación del Informe Coleman et al., (1966), constituye en punto de partida de estas investigaciones.

En este sentido, existen diversas visiones sobre el rendimiento académico que lo tratan como un fenómeno multifactorial (Edel-Navarro, 2003), o multicausal (Garbanzo, 2007), y en función de ello aparecen distintas variables explicativas. Destaca con un mayor impacto predictivo las calificaciones académicas procedentes de procesos de evaluación (Aitken, 1982; Toca \& Tourón, 1989; Jiménez, 1994; Miguel Díaz et al., 2002; Häkkinen, 2004; Rodríguez, Fita, \& Torrado, 2004; Ismail \& Othman, 2006, entre otros), la inteligencia (Cascón, 1995) o la riqueza sociocultural del contexto (Piñeros Jiménez \& Rodríguez Pinzón, 1998). Aitken (1982) descubre una significativa correlación entre la calificación de los exámenes de acceso y los resultados académicos de primer curso en Massachusetts. En un estudio realizado en la Universidad Carlos III de Madrid, García \& Segundo (2001) observan que la nota de acceso guarda una relación positiva, muy significativa, con el rendimiento académico tras acceder a la universidad. Según Cilliers \& Klerk (2001), las habilidades prácticas juegan también un papel predictivo importante en los resultados académicos.

El género, entre otros factores, es considerado por Di Gresia (2007) a través de un modelo tobit (Woolridge, 2010; Hill, Griffiths, \& Lim, 2012) en el que llega a que el desempeño es superior para las mujeres, para los estudiantes que ingresan más jóvenes a la universidad, para quienes provienen de hogares con padres más educados, y para los estudiantes que no trabajan, estudio realizado en la Facultad de Ciencias Económicas de la UN La Plata. Ismail \& Othman (2006); Barttolucci (1994); Valle \& Marcia (1995); Chaín Revueltas (1997); Velo Conde (2004); y Cortada de Kohan (2005), entre otros, también abordan el género como factor explicativo del rendimiento académico.

El idioma es también un factor importante en el rendimiento académico. Para los alumnos de primer curso de origen indio, frente a los de origen negro, en la universidad 
sudafricana de Johannesburgo (Stephen, Welman, \& Jordaan, 2004), el dominio de competencias en el idioma inglés fue un factor determinante. Asimismo, el programa de docencia en lengua inglesa de la Facultad de Turismo y Finanzas de la Universidad de Sevilla mostró mejores indicadores de rendimiento académico en porcentaje de no presentados, tasas de éxito, porcentaje de aprobados, distribución de calificaciones, notas medias por asignatura, expedientes medios y evolución de egresados que el de docencia en castellano (Valderas, Olmedo, \& Rodríguez, 2009).

En Oladokun, Adebanjo, \& Charles-Owaba (2008), un modelo de redes neuronales artificiales, mediante una combinación de factores, consigue predecir correctamente el rendimiento académico de más del 70 por ciento de estudiantes del Departamento de Ingeniería de la Universidad de Ibadan (Nigeria). Se usaron variables como resultados del examen de acceso, edad de ingreso, género, antecedentes de los padres, tipo y ubicación de la escuela secundaria a la que se asistió.

Observamos que en la literatura sobre rendimiento académico destaca la nota de acceso como variable predictora, no obstante, otras variables, como la que proponemos en este trabajo: opción de entrada en la carrera, pueden ayudar a describir mejor el desempeño académico.

En este sentido, un número importante de investigadores consideran que el ajuste de la titulación asignada con el deseo vocacional del estudiante predice el desempeño académico universitario. El orden de opción también es determinante. Así, autores como Salanova Soria, Martínez Martínez, Bresó Esteve, Llorens Gumbau, \& Grau Gumbau (2005) observan un mejor rendimiento académico en los estudiantes de la Universitat Jaume I que cursan sus primeras opciones de interés. También, en un estudio realizado en la Universidad de Barcelona por Rodríguez, Fita, \& Torrado (2004) y en la Universidad del País Vasco por Apodaha \& Gallareta (1999) se concluye con resultados similares.

Pero no estar en la carrera deseada no solo afecta al rendimiento académico sino también al abandono definitivo de la misma. Según Tinto (1995) el primer curso de carrera determina la evolución de los estudios, de manera que el $75 \%$ de estudiantes que no los termina aduce como principal motivo problemas en su primer año. Según Birch \& Miller (2007) el desempeño mostrado en el primer año también determina la duración en mayor o menor medida de los estudios.

Se observan escasos estudios sobre rendimiento académico en carreras de turismo, lo que confiere un valor añadido a nuestro trabajo. Lunar Leandro \& Marcano López (2007) en un estudio de la Escuela de Hostelería y Turismo de la Universidad de Oriente se limitan a constatar que en relación al perfil motivacional el 45,20\% de la población considera que la carrera realmente les gusta, y el $24,65 \%$ reconoce estar cursando la carrera por la facilidad de entrar en la universidad y luego cambiarse de carrera. No obstante, no estudian la correlación con el rendimiento académico.

Kwek, Bui, Rynne, \& So (2013) analizan el impacto de la autoestima y la resiliencia del estudiante en el rendimiento académico de estudiantes de primer curso en una escuela de Turismo y Hotelería de una universidad australiana. Los resultados sugieren, para los estudiantes internacionales y nacionales, que las dos variables estudiadas son predictores significativos del rendimiento académico. 
Frende Vega, Biedma Ferrer, \& Arana Jiménez (2017), a través de un análisis ANOVA, encuentran relación entre la percepción que tiene el estudiante sobre la asignatura Dirección de Recursos Humanos en Empresas Turísticas del Grado de Turismo de la Universidad de Cádiz y el rendimiento académico.

En definitiva, teniendo en cuenta que el rendimiento académico suele medirse mediante parámetros como las calificaciones de los estudiantes y la tasa de abandono, se hace necesario encontrar soluciones que ajusten satisfactoriamente la oferta de estudios a la demanda de los estudiantes. Ello debe contribuir positivamente a la formación de mejores egresados y beneficiará a la sociedad en su conjunto.

La literatura revisada coincide en aceptar que debe haber un parámetro que recoja el rendimiento académico que alcanzan los estudiantes universitarios. Según Tourón (1984), el rendimiento académico se expresa en una calificación cuantitativa y/o cualitativa que será el reflejo de un determinado aprendizaje, o de los logros pre-establecidos. De esta forma, las instituciones académicas y políticas disponen de un instrumento que debe favorecer la implementación de acciones encaminadas a la mejora tanto de la Educación Superior como de la asignación de recursos.

Se observa también que los estudios sobre la determinación de los factores que inciden en el rendimiento académico son cuantitativos, no siendo muy numerosos los que lo abordan desde una perspectiva cualitativa. Así, según Häkkinen (2004), las investigaciones cuantitativas, que utilizan principalmente análisis de correlación, concluyen que hasta el $25 \%$ del éxito futuro de los individuos se puede explicar a través de factores observables en la fecha en la que son admitidos en los estudios.

Por tanto, siguiendo los estudios observados y dado el carácter multidimensional del término "rendimiento académico" utilizaremos como indicador del mismo, las calificaciones obtenidas por el alumno, que constituyen un criterio que mide de forma objetiva el rendimiento del estudiante en el ámbito institucional.

El objetivo de nuestro trabajo es analizar y cuantificar el peso o influencia que tienen determinados factores observables personales e institucionales en el rendimiento académico -calificaciones- obtenido en el primer cuatrimestre (septiembre a enero) por los estudiantes del Grado en Turismo de la Facultad de Turismo y Finanzas de la Universidad de Sevilla. Para ello, se han utilizado como variables la nota de acceso y el sexo como determinantes personales del rendimiento académico; y como determinantes institucionales, el orden de preferencia por la titulación elegida, según el interés del estudiante, y la nota de corte de acceso a la titulación. La literatura existente sobre rendimiento académico soporta la utilización de estos factores como posibles determinantes del desempeño académico:

- Nota de acceso a la Universidad. Se destaca como uno de los predictores más importantes en el rendimiento académico junto al rendimiento académico previo a la Universidad en estudios sobre este concepto en instituciones de educación superior. Este factor está ligado a la nota de corte, como factor institucional, para acceder a las titulaciones universitarias ofertadas (Garbanzo, 2007).

- Sexo. Consideramos esta variable en la cohorte objeto de estudio, puesto que facilita información relevante sobre la existencia de posibles comportamientos diferenciales en hombres y mujeres. Algunos investigadores (Montero Rojas, Villalobos 
Palma, \& Valverde Bermúdez, 2007) señalan que, además de los rasgos innatos diferenciales entre hombres y mujeres, las pautas de socialización y el refuerzo de aptitudes diferenciales por sexo justifican posibles diferencias en el rendimiento académico de hombres y mujeres.

- Orden de preferencia por la titulación. Esta variable hace referencia a la opción ordinal expresada por el estudiante para acceder a la titulación, pudiendo ser su primera elección o, no habiéndola elegido como primera opción, ser asignado a la titulación por la capacidad de asignación de cupos que tiene la propia Universidad (Garbanzo, 2007). Un número importante de estudios empíricos sobre rendimiento académico destacan que realizar una carrera universitaria en primera opción afecta decisivamente en el rendimiento académico del estudiante, sobre todo en los primeros resultados obtenidos.

- Nota de corte para el acceso a la titulación. Como factor institucional, esta variable es de gran importancia en la toma de decisiones, ya que se puede controlar o modificar para incidir de forma directa en el rendimiento académico. En muchas universidades se determina un límite máximo de ingreso de estudiantes en primer curso por motivos de capacidad espacial o metodológica, que actúa indirectamente como nota de corte. En general, se ha comprobado que los países que realizan procesos de selección más restrictivos en cuanto a los requisitos de entrada presentan un nivel de fracaso más bajo entre los estudiantes de primer año (Toca \& Tourón, 1989).

\section{METODOLOGÍA}

\subsection{Justificación del instrumento de investigación}

Se pretende modelizar las calificaciones en las diferentes disciplinas impartidas en el primer cuatrimestre de primer curso del Grado en Turismo, en función de variables que podrían ser consideradas como explicativas de las mismas como son el sexo y la calificación de acceso a la universidad (que tiene un rango entre 5 y 14 puntos). Los modelos de regresión nos permiten estimar el efecto marginal de esas variables explicativas o regresoras sobre las calificaciones que son objeto de modelización.

El método de Mínimos Cuadrados Ordinarios (MCO) estima la media de la variable objetivo condicionada a los valores de las regresoras, y exige unas hipótesis iniciales sobre la relación entre explicativas, predictivas o regresoras, y la variable objetivo. El resultado de la estimación es una consecuencia de una minimización de suma de errores al cuadrado, que proporciona una media condicionada como valor óptimo. Así, por ejemplo, si disponemos de dos variables predictivas, $X_{1}$ y $X_{2}$, y una variable objetivo $Y$, planteamos el modelo lineal $y_{i}=\alpha+\beta_{1} \cdot x_{1 i}+\beta_{2} \cdot x_{2 i}+u_{i} ; \quad i=1, \ldots, n$, donde $x_{1 i}, x_{2 i}$ e $y_{i}$ son los correspondientes valores de las tres variables implicadas en la observación, para el caso del individuo $i$ de una muestra de tamaño $n$, y $\alpha, \beta_{1}$ y $\beta_{2}$ (intercepto y pendientes, son los parámetros a estimar), y $u_{i}$ es una variable no observada de carácter aleatorio conocida como "perturbación aleatoria" en un contexto econométrico y que garantiza la igualdad planteada en el modelo. El método consiste en resolver el problema de optimización $\underset{\beta_{1}, \beta_{2}}{\operatorname{Min}} \sum_{i=1}^{n}\left(y_{i}-\alpha-\beta_{1} x_{1 i}-\beta_{2} x_{2 i}\right)^{2}$. 
El resultado de esa optimización es la estimación de la media de la variable objetivo condicionada a los valores observados de las variables explicativas, o sea, $\hat{E}\left(y_{i} \mid x_{1 i}, x_{2 i}\right)=\hat{\alpha}+\hat{\beta}_{1} \cdot x_{1 i}+\hat{\beta}_{2} \cdot x_{2 i} ; i=1, \ldots, n$. El proceso de estimación exige unas condiciones a las perturbaciones aleatorias $u_{i}$ que intervienen en el modelo. Hemos de añadir que en muchas estadísticas aplicadas la elaboración de modelos de regresión y la aplicación de métodos de estimación del tipo mínimos cuadrados ordinarios es algo habitual, aunque a veces, se nos antoja, puede ser incompleta. Ya Mosteller \& Tukey (1977), en su influyente texto, señalan:

Lo que la curva de regresión hace es dar un gran resumen de los promedios de las distribuciones correspondientes al conjunto de $X$ s. Podríamos ir más lejos y calcular varias curvas de regresión diferentes correspondientes a los diferentes puntos porcentuales de las distribuciones y así obtener una imagen más completa del conjunto. Normalmente esto no se hace, y por lo tanto la regresión a menudo da una imagen bastante incompleta. Así como la media da una imagen incompleta de una sola distribución, por lo que la curva de regresión da una imagen correspondiente incompleta para un conjunto de distribuciones.

¿Por qué la estimación por mínimos cuadrados del modelo de regresión lineal aparece tanto en las estadísticas aplicadas? ¿Qué hace que ese método sea una herramienta exitosa? Dos posibles respuestas se sugieren. El hecho de que los estimadores proporcionados por el método MCO sean lineales proporciona una comodidad muy atractiva. Seguramente este fue el impulso inicial para su éxito. Y en segundo lugar, si las perturbaciones del modelo se distribuyen normalmente, se sabe que los métodos de los mínimos cuadrados gozan de cierta optimalidad.

Y, sin embargo, como los mismos Mosteller \& Tukey (1977) sugieren, la media rara vez es un fin satisfactorio en sí mismo, incluso para el análisis estadístico de una sola muestra. Medidas de dispersión, de asimetría, de curtosis, diagramas de cajas, histogramas y estimaciones de funciones de densidad más sofisticadas se emplean frecuentemente para obtener mayor información. ¿Puede algo similar hacerse en la regresión? Una forma de generalizar la regresión sería complementar la media condicionada estimada por mínimos cuadrados con estimaciones de varios cuantiles condicionados. $\mathrm{O}$ sea, manejar regresiones cuantiles en las que otros puntos distintos a la media, y localizados, son modelizados de manera condicionada al estilo de la propia media. Es lo que se pretende en este trabajo.

Hemos comentado que las técnicas de estimación MCO exigen unas condiciones relacionadas con la aleatoriedad de las perturbaciones que se pueden resumir en $u_{i} \approx N\left(0, \sigma^{2}\right)$. La técnica de regresión cuantílica no necesita de tales hipótesis para la estimación de parámetros, no considerando ninguna restricción sobre las perturbaciones. Se suele clasificar este método como semiparamétrico, en el que se establece un tipo de relación entre los regresores y la endógena sin incluir ninguna hipótesis sobre la perturbación aleatoria. Como ya se ha apuntado, el método de estimación mínimo cuadrático tiene por objetivo minimizar la suma de los residuos al cuadrado; en la regresión cuantílica el objetivo es minimizar una suma de errores absolutos ponderados con pesos asimétricos. Un caso especial de la regresión cuantílica será la regresión mediana, en la cual los pesos son simétricos y la regresión tendrá por objetivo minimizar la suma de las desviaciones en términos absolutos sin ponderar. 
El hecho de no conocer y no considerar la distribución del término de error tiene el inconveniente de no poder analizar las propiedades de los estimadores, limitando el proceso de inferencia. Pero a su vez tiene la ventaja de no estar sujeto a tales hipótesis cuando, como es frecuente, éstas no se cumplen, caso de no normalidad, asimetría y/o heterocedasticidad. Además la regresión cuantílica permite un conocimiento de la distribución condicionada cuando ésta varía para diferentes niveles o valores de la variable explicativa $X$, dependiendo de la ponderación que se establezca. Según Buchinsky (1995) existen múltiples aplicaciones con regresiones cuantílicas y en campos muy diversos, pero en general se obtienen mejores resultados, frente a los procedimientos convencionales, cuando se dispone de una gran cantidad de datos de corte transversal. En estos casos, la información disponible no suele acomodarse a las fuertes restricciones impuestas en las hipótesis básicas del modelo de regresión lineal, y sus problemas de heterocedasticidad, de no normalidad y/o de asimetría son habituales. En Koenker \& Hallock (2001) se detallan las ventajas de la estimación cuantílica frente a métodos más tradicionales, resaltando los casos en los que los incumplimientos de determinadas hipótesis conducen a resultados más fiables con el empleo de la regresión cuantílica.

Aunque el método de regresión cuantil tiene sus inicios a finales de los años setenta con el trabajo de Koenker \& Basset (1978), el propio Koenker (2005) afirma que la idea básica de la que parte la regresión cuantílica se encuentra en los trabajos de Boskovich de la segunda mitad del siglo XVIII, acerca del estudio de la forma elíptica de la Tierra; en ellos, Boskovich empieza a usar la minimización del valor absoluto de los residuos para encontrar los parámetros de la función de la elíptica de la Tierra. Más tarde Laplace y Edgeworth investigarían sobre esta técnica, al igual que Koenker y Basset harían alrededor de un siglo después, para estimar los parámetros de la regresión cuantílica. Por tanto, las primeras ideas que pueden asociarse con la regresión cuantílica datan de fechas anteriores al nacimiento de los mínimos cuadrados de Adrien-Marie Legendre en 1805.

\subsection{El modelo de regresión}

En general, para un modelo del tipo $y_{i}=\alpha+\beta_{1} \cdot x_{1 i}+\beta_{2} \cdot x_{2 i}+u_{i} ; i=1, \ldots, n$ (usamos dos variables explicativas como ejemplo ilustrativo) la estimación de un cuantil $\tau$ (con $0 \leq \tau \leq 1)$ de la variable objetivo $Y$ condicionada a los valores de las variables explicativas $X_{1}$ y $X_{2}$ consiste en resolver:

$$
\operatorname{Min}_{\beta_{1}, \beta_{2}}\left[\sum_{y_{i} \geq \alpha+\beta_{1} x_{1 i}+\beta_{2} x_{2 i}} \tau\left|y_{i}-\alpha-\beta_{1} x_{1 i}-\beta_{2} x_{2 i}\right|+\sum_{y_{i}<\alpha+\beta_{1} x_{1 i}+\beta_{2} x_{2 i}}(1-\tau)\left|y_{i}-\alpha-\beta_{1} x_{1 i}-\beta_{2} x_{2 i}\right|\right],
$$

donde los valores obtenidos para $\alpha, \beta_{1}, \beta_{2}$ que minimicen esa suma ponderada son las estimaciones cuantiles del modelo. Obviamente, si $\tau=0$ ' 5 obtenemos la estimación de la mediana de $Y$ condicionada a los valores de las variables explicativas. Normalmente, los resultados óptimos se suelen obtener mediante métodos iterados de optimización. Al igual que en MCO, el valor que minimiza la suma de residuos al cuadrado es la media condicional de $Y$ dadas las explicativas, y ahora el que minimiza esas sumas en valor absoluto es el cuantil condicional de $Y$ dadas las mismas variables. 
Como ya se ha puesto de manifiesto, lo que se lleva a cabo, en este momento, es una minimización de las desviaciones absolutas ponderadas con pesos asimétricos; es decir, que a cada desviación correspondiente a la observación $i$ se le da más o menos peso según el cuantil cuya recta de regresión se esté estimando. La principal ventaja que aporta el uso de las desviaciones en valor absoluto, en lugar de las desviaciones al cuadrado, es el comportamiento ante la existencia de valores atípicos. Ante tal situación, la estimación que ofrece la regresión cuantílica prácticamente no se ve alterada por valores extremos, ya que "penaliza" los errores de forma lineal; mientras que la regresión MCO, al elevar los errores al cuadrado, lo que hace es darle mayor importancia precisamente a dichos valores, "penalizándolos" de forma cuadrática.

Respecto a la introducción de ponderaciones asimétricas, dado que el objetivo es estimar varias rectas de regresión que pasen por distintos puntos de la distribución, el papel que cumplen los pesos asimétricos es precisamente el de situar esas rectas ponderando de forma distinta los residuos positivos y los negativos.

$\mathrm{Si}$, por ejemplo, estuviésemos interesados en estimar la recta correspondiente al cuantil 80, que uniría los puntos del cuantil 80 de la distribución condicionada de $Y$ dadas $X_{1}$ y $X_{2}$, para su estimación, los residuos positivos (cuando el valor real es mayor que el estimado) se ponderan con 0,80 ; mientras que los negativos (cuando el valor real es menor que el estimado) se ponderan con 0,20. Como ejemplo, supongamos que el salario de una población varía según sea su nivel de formación, pero que la elasticidad y la pendiente son diferentes en el extracto de salarios altos, pues su aumento es más elevado que el que le corresponde por el nivel medio. La regresión por mínimos cuadrados encontrará la respuesta media en términos de pendiente y no diferenciará la existencia de un cambio en el parámetro. Por el contrario, una regresión cuantílica, revelará que en los cuantiles superiores el parámetro aumenta de tamaño. Podría argumentarse que una segmentación de la muestra y su posterior estimación por mínimos cuadrados en cada segmento nos llevarían a la misma conclusión, pero ello nos podría generar un sesgo de selección como señala Heckman (1979). La ventaja que aporta la regresión cuantílica frente a esta estrategia es que en cada cuantil intervienen todas las observaciones convenientemente ponderadas.

Como ya se ha dicho, el procedimiento de estimación cuantil es de tipo semiparamétrico, sin establecimiento de hipótesis sobre el error. Eso hace que el cálculo directo de la significatividad de los parámetros y el desarrollo de los contrastes de nulidad resulte más complicado que en la regresión mínimo cuadrática.

La literatura ofrece diferentes soluciones y en el trabajo de Koenker \& Hallock (2001) se presentan los resultados de una simulación con cinco procedimientos alternativos: Intervalos de inversión de rangos de Koenker, Ng, \& Portnoy (1994), el método Sandwich de Hasan \& Koenker (1997), el método de Sandwich de Powell (1989), el estimador Siddiqui de la covarianza basado en errores independiente e idénticamente distribuidos (errores iid) y tres versiones de aleatorización muestral o "bootstrap" (20, 200, 600). En el trabajo mencionado no encuentran problemas especiales en ninguno de ellos y sus conclusiones son similares.

Además de estos procedimientos hay que resaltar el procedimiento clásico "Sandwich" de Huber (1967) que está basado en errores que no son independientes e idénticamente distribuidos (iid) y que es recogido por programas como SAS o EVIEWS. Por 
su parte STATA también calcula estimaciones asintóticas del error basándose en errores iid e incluye el método de Koenker \& Basset (1978), y Koenker \& Hallock (2001).

En nuestro caso, hemos calculado errores estándar con estimaciones robustas respecto a la heterocedasticidad usando el método de Koenker \& Zhao (1994). Para la construcción de intervalos de confianza se usa el método de inversión de rango para los que, en general, los intervalos pueden resultan asimétricos respecto a las estimaciones puntuales. Los usados aquí se basan en el estimador robusto de Koenker \& Machado (1999).

\subsection{Muestra y variables analizadas}

Sobre los datos obtenidos del Servicio de Informática y Comunicaciones (SIC) de la Universidad de Sevilla, se ha tomado una muestra de 572 alumnos/as de nuevo ingreso en el Grado en Turismo, de la Facultad de Turismo y Finanzas de la Universidad de Sevilla. Corresponde a los alumnos que ingresaron en el curso 2014-15 (una muestra de tamaño 295 a la que llamamos cohorte 1) y a los que ingresaron en el curso siguiente, 2015-16 (una muestra de tamaño 277, llamada cohorte 2). Los 18 alumnos de diferencia entre ambas cohortes hacen que la nota mínima de acceso a estos estudios haya cambiado de 5'10 en la primera a 5'42 en la segunda. Las variables analizadas son las que siguen:

Nota: Calificación de acceso a la universidad, con un rango entre 5,00 y 14,00.

Sexo: que toma los valores 0: Mujer y 1: Hombre.

ECO_1: Calificación obtenida en la asignatura "Economía I", con un rango entre 0,00 у 10,00 .

ETMA: Calificación obtenida en la asignatura "El Turismo en el Mundo Actual", con un rango entre 0,00 y 10,00 .

EyOT: Calificación obtenida en la asignatura "Empresas y Organizaciones Turísticas" con un rango entre 0,00 y 10,00 .

FdC: Calificación obtenida en la asignatura "Fundamentos de Contabilidad", con un rango entre 0,00 y 10,00 .

IaF: Calificación obtenida en la asignatura "Introducción a las Finanzas", con un rango entre 0,00 y 10,00 .

También incorporamos la variable dicotómica Curso, que toma el valor 1 si el alumno ingresó en 2014-15 (si pertenece a la primera cohorte) y 2 si ingresó en 2015-16 (si pertenece a la segunda cohorte), y la variable Orden, que nos indica el orden de preferencia en el que se encontraba el Grado en Turismo cuando el alumno solicitó hacer estudios universitarios, y que toma el valor 1 si el Grado en Turismo fue elegido en primer lugar, 2, si fue elegido en segundo lugar, ... 5 si fue elegido en quinto lugar o un lugar más retrasado aún.

\section{RESULTADOS}

A continuación se muestran la distribución de frecuencias y los principales estadísticos de las variables objeto de estudio de los estudiantes en sus primeros cuatro meses de estudios universitarios. 
En primer lugar, en la Tabla 1 se expone la distribución de frecuencias de la variable ORDEN, para la muestra completa y para cada cohorte por separado. Destaca que más del $75 \%$ de los estudiantes de nuevo ingreso ha elegido su carrera en primer o segundo lugar, lo que, en principio, debe interpretarse como un signo positivo, pues debería tratarse de personas más motivadas.

Tabla 1

DISTRIBUCIÓN DE FRECUENCIAS DEL ORDEN DE ELECCIÓN DEL GRADO EN TURISMO

\begin{tabular}{llll}
\hline Orden de elección & $\begin{array}{l}\text { Número de alumnos } \\
\text { Muestra completa }\end{array}$ & $\begin{array}{l}\text { Número de alumnos } \\
\text { Curso 2014-15 }\end{array}$ & $\begin{array}{l}\text { Número de } \\
\text { alumnos } \\
\text { Curso 2015-16 }\end{array}$ \\
\hline 1 & $370\left(6^{\prime} 4^{\prime} 69 \%\right)$ & $183\left(62^{\prime} 03 \%\right)$ & $187\left(67^{\prime} 51 \%\right)$ \\
2 & $69\left(1^{\prime}, 06 \%\right)$ & $41\left(13^{\prime} 90 \%\right)$ & $28\left(10^{\prime} 11 \%\right)$ \\
3 & $54\left(9^{\prime} 44 \%\right)$ & $32\left(10^{\prime} 85 \%\right)$ & $22\left(7^{\prime} 94 \%\right)$ \\
4 & $28\left(4^{\prime} 90 \%\right)$ & $13\left(4^{\prime} 41 \%\right)$ & $15\left(5^{\prime} 42 \%\right)$ \\
5 o más & $51\left(8^{\prime} 92 \%\right)$ & $26\left(8^{\prime} 81 \%\right)$ & $25\left(9^{\prime} 03 \%\right)$ \\
\hline Total & $\mathbf{5 7 2}$ & $\mathbf{2 9 5}$ & $\mathbf{2 7 7}$ \\
\hline
\end{tabular}

Seguidamente, la distribución de frecuencias de la variable sexo, para las tres situaciones antes expuestas, aparece en la Tabla 2. Aquí cabe resaltar que más de dos tercios del estudiantado son mujeres, hecho que suele repetirse en carreras de turismo.

Tabla 2

DISTRIBUCIÓN DE FRECUENCIAS DEL SEXO DE ALUMNOS MATRICULADOS EN GRADO EN TURISMO

\begin{tabular}{llll}
\hline Sexo & $\begin{array}{l}\text { Número de alumnos } \\
\text { Muestra completa }\end{array}$ & $\begin{array}{l}\text { Número de alumnos } \\
\text { Curso 2014-15 }\end{array}$ & $\begin{array}{l}\text { Número de alumnos } \\
\text { Curso 2015-16 }\end{array}$ \\
\hline Mujer & $380\left(66^{\prime} 43 \%\right)$ & $197\left(66^{\prime} 78 \%\right)$ & $183\left(66^{\prime} 06 \%\right)$ \\
Hombre & $192\left(33^{\prime} 57 \%\right)$ & $98\left(33^{`} 22 \%\right)$ & $94\left(33^{\prime} 94 \%\right)$ \\
\hline Total & $\mathbf{5 7 2}$ & $\mathbf{2 9 5}$ & $\mathbf{2 7 7}$ \\
\hline
\end{tabular}

Las Tablas 3, 4 y 5 muestran los estadísticos principales asociados a las variables cuantitativas que forman parte de nuestro análisis: la Tabla 3, para la muestra completa, la 4 para la primera cohorte, y la 5 para la segunda cohorte. 
Tabla 3

ESTADÍSTICOS PRINCIPALES, USANDO LA MUESTRA COMPLETA

\begin{tabular}{lllll}
\hline Variable & Media & Mediana & Mínimo & Máximo \\
\hline NOTA & 7.30837 & 7.08500 & 5.10 & 11.85 \\
ECO_I & 6.10826 & 6.00000 & 0.00 & 10.00 \\
ETMA & 6.95183 & 7.00000 & 1.45 & 10.00 \\
EyOT & 5.95909 & 6.10000 & 1.30 & 10.00 \\
FdC & 4.53975 & 4.34973 & 0.00 & 9.30 \\
IaF & 5.13460 & 5.05000 & 0.00 & 10.00 \\
\hline Variable & Desv. Típica & Coef. Variación & Asimetría & Curtosis \\
\hline NOTA & 1.29227 & 0.176820 & 0.988630 & 0.965525 \\
ECO_I & 1.92517 & 0.315175 & -0.635185 & 0.322885 \\
ETMA & 1.41831 & 0.204019 & -0.922701 & 2.20222 \\
EyOT & 1.84389 & 0.309425 & -0.214183 & -0.422424 \\
FdC & 1.61467 & 0.355673 & 0.269147 & 0.361367 \\
IaF & 2.14005 & 0.416790 & -0.207722 & -0.233232 \\
\hline Variable & Percentil 5 & Percentil 95 & Rango & \\
\hline NOTA & 5.583 & 10.043 & Intercuartílico & \\
ECO_I & 2.000 & 9.000 & 1.585 & \\
ETMA & 4.365 & 9.000 & 2.800 & \\
EyOT & 2.700 & 9.000 & 1.500 & \\
FdC & 1.965 & 7.500 & 2.200 & \\
IaF & 1.332 & 8.550 & 2.350 & \\
\hline & & & & \\
\hline
\end{tabular}

Tabla 4

ESTADÍSTICOS PRINCIPALES, USANDO LA PRIMERA COHORTE

\begin{tabular}{lllll}
\hline Variable & Media & Mediana & Mínimo & Máximo \\
\hline NOTA & 7.30986 & 7.04000 & 5.10000 & 11.6800 \\
ECO_I & 6.02283 & 6.00000 & 0.00000 & 10.0000 \\
ETMA & 6.92507 & 7.00000 & 1.50000 & 10.0000 \\
EyOT & 5.31501 & 5.39248 & 1.30000 & 9.80000 \\
FdC & 4.27041 & 4.22998 & 0.00000 & 9.30000 \\
IaF & 4.62535 & 4.69838 & 0.00000 & 10.0000 \\
\hline Variable & Desv. Típica & Coef. Variación & Asimetría & Curtosis \\
\hline NOTA & 1.30101 & 0.177980 & 1.01771 & 1.00469
\end{tabular}




\begin{tabular}{lllll} 
ECO_I & 1.95133 & 0.323989 & -0.674510 & 0.491669 \\
ETMA & 1.32443 & 0.191252 & -0.969856 & 2.99421 \\
EyOT & 1.76586 & 0.332241 & -0.0308416 & -0.439226 \\
FdC & 1.49795 & 0.350775 & 0.466513 & 1.97746 \\
IaF & 1.98708 & 0.429606 & 0.0194406 & 0.273516 \\
\hline Variable & Percentil 5 & Percentil 95 & Rango & \\
\hline NOTA & 5.52000 & 10.1020 & 1.45000 & \\
ECO_I & 2.00000 & 9.00000 & 2.00000 & \\
ETMA & 4.00000 & 9.00000 & 1.10000 & \\
EyOT & 2.30000 & 8.30000 & 2.60000 & \\
FdC & 1.68000 & 7.34000 & 0.335788 & \\
IaF & 0.800000 & 8.00000 & 2.20000 & \\
\hline
\end{tabular}

Tabla 5

ESTADÍSTICOS PRINCIPALES, USANDO LA SEGUNDA COHORTE

\begin{tabular}{lllll}
\hline Variable & Media & Mediana & Mínimo & Máximo \\
\hline NOTA & $\mathbf{7 . 3 0 6 7 9}$ & 7.09000 & $\mathbf{5 . 4 2 0 0 0}$ & 11.8500 \\
ECO_I & 6.19924 & 6.80000 & 0.800000 & 9.90000 \\
ETMA & 6.98034 & 6.95000 & 1.45000 & 10.0000 \\
EyOT & 6.64502 & 6.63854 & 1.80000 & 10.0000 \\
FdC & 4.82659 & 4.88326 & 0.900000 & 9.20000 \\
IaF & 5.67695 & 5.63940 & 0.00000 & 10.0000 \\
\hline Variable & Desv. Típica & Coef. Variación & Asimetría & Curtosis \\
\hline NOTA & 1.28525 & 0.175898 & 0.956295 & 0.919958 \\
ECO_I & 1.89620 & 0.305876 & -0.584912 & 0.0893347 \\
ETMA & 1.51379 & 0.216865 & -0.892766 & 1.58140 \\
EyOT & 1.67233 & 0.251666 & -0.421977 & 0.0118212 \\
FdC & 1.68626 & 0.349369 & 0.0326296 & -0.549436 \\
IaF & 2.16715 & 0.381745 & -0.551700 & -0.125832 \\
\hline Variable & Percentil 5 & Percentil 95 & Rango & \\
\hline NOTA & 5.61900 & 9.66600 & 1.71500 & \\
ECO_I & 2.80000 & 9.00000 & 2.80000 & \\
ETMA & 4.45000 & 9.45000 & 1.50000 & \\
EyOT & 3.60000 & 9.21000 & 2.00000 & \\
FdC & 2.09000 & 7.60000 & 2.30000 & \\
IaF & 1.45000 & 8.85000 & 2.35000 & \\
\hline
\end{tabular}


Observamos la casi absoluta igualdad de la nota media de acceso de ambas cohortes (el valor de $p$ del contraste de comparación de medias es 0’9773), que está por encima de 7,30. La principal diferencia entre ambas es la calificación mínima, como ya se ha comentado. Creemos importante subrayar que la media expresa mayor información del nivel académico de estos estudiantes, que el dato de la nota de corte (nota más baja con la que ingresó el último estudiante), que es la que aparece públicamente en los medios. Esta última suele situarse entre 5 y 6 en carreras de turismo, y es interpretada, en numerosas ocasiones, como la nota con la que accede la mayor parte de los estudiantes, lo que en nuestro caso, no es cierto. Sin embargo, esta nota de corte sí nos interesa para nuestra investigación. En la primera cohorte fue de 5'1 mientras que en la segunda de 5'42, provocado sin duda por una reducción en el número de estudiantes que podían acceder a estos estudios. ¿Ese cambio de valor mínimo genera un cambio en la pendiente asociada al cuantil (en este caso del 5\%) más bajo de cada una de las calificaciones?

Asimismo, se han llevado a cabo los contrastes de normalidad (en total cuatro contrastes clásicos de normalidad) para las cinco variables objetivo y para las dos cohortes, y los mismos rechazan la hipótesis de normalidad en todos los casos, una de las hipótesis exigidas en la estimación por MCO. Esto significa que la hipótesis de normalidad exigida por el método MCO no se cumple. La tabla 6 recoge los valores $p$ asociados a dichos contrastes.

Los percentiles 5 y 95 nos sitúan en las colas de la distribución de cada una de las calificaciones. El primero nos define las calificaciones más bajas (por debajo de su valor sólo hay un 5\% del total de calificaciones), y el segundo nos sitúa en el lugar donde se encuentran las calificaciones más elevadas (por encima sólo hay un 5\%).

Tabla 6

VALORES P DE LOS CONTRASTES DE NORMALIDAD DE LAS CINCO VARIABLES OBJETIVO PARA LAS DOS COHORTES

\begin{tabular}{|c|c|c|c|c|c|}
\hline & \multirow[b]{2}{*}{ Asignatura } & \multicolumn{4}{|c|}{ Contrastes de normalidad. Valor $p$} \\
\hline & & Doornik-Hansen & W de Shapiro-Wilk & Lilliefors & Jarque-Bera \\
\hline \multirow{5}{*}{$\begin{array}{l}\text { Primera } \\
\text { cohorte }\end{array}$} & ECO_I & $3.33 \mathrm{e}-006$ & $1.08 \mathrm{e}-009$ & 0.0000 & $3.143 \mathrm{e}-006$ \\
\hline & ETMA & $7.88 \mathrm{e}-009$ & $1.96 \mathrm{e}-011$ & 0.0000 & $1.067 \mathrm{e}-034$ \\
\hline & EyOT & 0.003452 & 0.0002287 & 0.0000 & 0.0029848 \\
\hline & FdC & $6.27 \mathrm{e}-007$ & $1.53 \mathrm{e}-013$ & 0.0000 & $1.735 \mathrm{e}-013$ \\
\hline & $\mathbf{I a F}$ & 0.004313 & $2.05 \mathrm{e}-008$ & 0.0000 & 0.0062558 \\
\hline \multirow{5}{*}{$\begin{array}{l}\text { Segunda } \\
\text { cohorte }\end{array}$} & ECO_I & $1.70 \mathrm{e}-005$ & $2.44 \mathrm{e}-007$ & 0.0000 & 0.0003555 \\
\hline & ETMA & $1.30 \mathrm{e}-007$ & $2.51 \mathrm{e}-009$ & 0.0000 & $5.527 \mathrm{e}-015$ \\
\hline & EyOT & 0.006849 & 0.000356 & 0.0000 & 0.0163894 \\
\hline & FdC & 0.017226 & 0.000527 & 0.0000 & 0.0170901 \\
\hline & $\mathbf{I a F}$ & $7.19 \mathrm{e}-006$ & $4.29 \mathrm{e}-008$ & 0.0000 & 0.00081095 \\
\hline
\end{tabular}




\subsection{Modelo especificado}

En todos los casos, el modelo propuesto para estimar es el que sigue:

Asignatura $_{y}=\alpha+\beta_{1} \cdot$ Nota $_{i}+\beta_{2} \cdot$ Sexo $_{i}+\beta_{3} \cdot$ Orden $_{i}+u_{i}$, con $j=1,2, . ., 5$ representa cada una de las cinco asignaturas cuyas calificaciones son variables objetivo en nuestro modelo, y con $i=1, \ldots, 295$ representando a cada uno de los individuos de la muestra de la primera cohorte, y $i=1, \ldots, 277$, para la segunda cohorte. Por tanto, al haber cinco variables objetivo estimamos cinco modelos de regresión, en cada una de las dos cohortes. Vamos a utilizar MCO, para estimar las medias condicionadas, como se ha expuesto, y emplearemos la regresión cuantil estimando cuantiles estratégicos (0’05, 0’25, 0’50, 0'75 y 0'95) de las variables objetivo, repartidos a lo largo del rango de las mismas. Posteriormente, iremos comparando las estimaciones de ambas cohortes en cada uno de los casos. Con ello, observaremos el valor y la significación de las pendientes en cada uno de los modelos estimados, y podremos comparar el peso o influencia que tienen las variables explicativas sobre el valor medio, así como sobre los cuartiles de las variables objetivos.

\section{Tabla 7 \\ ANÁLISIS DE REGRESIÓN MCO, ECO_I}

\begin{tabular}{lcclll}
\hline & Coeficiente & Desv. Típica & Estadístico t & \multicolumn{1}{l}{ Valor p } & \\
\hline Modelo 1. Observaciones 1-295. Primera cohorte .Variable dependiente: ECO_I & \\
\hline const & 4.57602 & 0.738151 & 6.1993 & $<0.0001$ & $* * *$ \\
NOTA & 0.180861 & 0.0905846 & 1.9966 & 0.0468 & $* *$ \\
SEXO & -0.0673378 & 0.245488 & -0.2743 & 0.7840 & \\
ORDEN & 0.0799242 & 0.0899115 & 0.8889 & 0.3748 & \\
\hline Modelo 2. Observaciones 1-277. Segunda cohorte .Variable dependiente: ECO_I & \\
\hline const & 3.95773 & 0.678998 & 5.8288 & $<0.0001$ & $* * *$ \\
NOTA & 0.330004 & 0.0869981 & 3.7932 & 0.0002 & $* * *$ \\
SEXO & -0.245007 & 0.235589 & -1.0400 & 0.2993 & \\
ORDEN & -0.0485704 & 0.0845341 & -0.5746 & 0.5661 & \\
\hline
\end{tabular}

Se aprecia en la tabla 7 que en ambas cohortes, para el caso de la estimación por mínimos cuadrados ordinarios (MCO), el SEXO y el ORDEN no son variables significativas. La variable NOTA de acceso a la universidad sí lo es, con una relación directa o positiva, y la pendiente es mayor en la segunda (0'33 frente a 0'18).

Efectuamos a continuación las estimaciones mediante regresión cuantil. 


\section{Tabla 8}

ANÁLISIS DE REGRESIÓN CUANTIL, ECO_I

\begin{tabular}{|c|c|c|c|c|}
\hline & tau & Coeficiente & Desv. Típica & Estadístico t \\
\hline \multicolumn{5}{|c|}{ Modelo 3. Observaciones 1-295. Primera cohorte Variable dependiente: ECO_I } \\
\hline \multirow[t]{5}{*}{ const } & 0.050 & 2.00000 & 3.25949 & 0.613592 \\
\hline & 0.250 & 5.00000 & 1.23598 & 4.04538 \\
\hline & 0.500 & 4.82124 & 0.550543 & 8.75726 \\
\hline & 0.750 & 5.11302 & 0.777742 & 6.57419 \\
\hline & 0.950 & 9.00000 & 1.30924 & 6.87419 \\
\hline \multirow[t]{5}{*}{ NOTA } & 0.050 & $3.09461 \mathrm{e}-011$ & 0.388882 & $7.95772 \mathrm{e}-011$ \\
\hline & 0.250 & $2.86955 \mathrm{e}-010$ & 0.152698 & $1.87923 \mathrm{e}-009$ \\
\hline & 0.500 & 0.188324 & 0.0772398 & 2.43817 \\
\hline & 0.750 & 0.299401 & 0.0662989 & 4.51593 \\
\hline & 0.950 & $-3.47214 \mathrm{e}-015$ & 0.158135 & $-2.19569 \mathrm{e}-014$ \\
\hline \multirow[t]{5}{*}{ SEXO } & 0.050 & $7.86410 \mathrm{e}-011$ & 1.33371 & $5.89643 \mathrm{e}-011$ \\
\hline & 0.250 & $2.28650 \mathrm{e}-010$ & -0.343811 & $-6.65045 \mathrm{e}-010$ \\
\hline & 0.500 & -0.0843834 & 0.161321 & -0.523077 \\
\hline & 0.750 & 0.119012 & 0.345726 & 0.344238 \\
\hline & 0.950 & $1.71057 \mathrm{e}-012$ & 0.431249 & $3.96654 \mathrm{e}-012$ \\
\hline \multirow[t]{5}{*}{ ORDEN } & 0.050 & $3.57716 \mathrm{e}-011$ & 0.591631 & $6.04626 \mathrm{e}-011$ \\
\hline & 0.250 & $1.87286 \mathrm{e}-010$ & 0.0985217 & $1.90096 \mathrm{e}-009$ \\
\hline & 0.500 & -0.00956783 & 0.0753441 & -0.126988 \\
\hline & 0.750 & -0.0321856 & 0.123547 & -0.260512 \\
\hline & 0.950 & $1.09272 \mathrm{e}-013$ & 0.238493 & $4.58179 \mathrm{e}-013$ \\
\hline \multicolumn{5}{|c|}{ Modelo 4. Observaciones 1-277. Segunda cohorte Variable dependiente: ECO_I } \\
\hline \multirow[t]{5}{*}{ const } & 0.050 & -3.01061 & 1.97325 & -1.52571 \\
\hline & 0.250 & 2.12826 & 0.773013 & 2.75320 \\
\hline & 0.500 & 4.69675 & 0.826496 & 5.68273 \\
\hline & 0.750 & 5.00479 & 0.662240 & 7.55737 \\
\hline & 0.950 & 6.71107 & 0.539293 & 12.4442 \\
\hline \multirow[t]{5}{*}{ NOTA } & 0.050 & 0.757576 & 0.303912 & 2.49274 \\
\hline & 0.250 & 0.434783 & 0.0665383 & 6.53432 \\
\hline & 0.500 & 0.282955 & 0.110453 & 2.56177 \\
\hline & 0.750 & 0.354610 & 0.0858434 & 4.13089 \\
\hline & 0.950 & 0.331325 & 0.0457565 & 7.24105 \\
\hline \multirow[t]{5}{*}{ SEXO } & 0.050 & -0.339394 & 0.732572 & -0.463291 \\
\hline & 0.250 & 0.00869565 & 0.252280 & 0.0344682 \\
\hline & 0.500 & -0.376602 & 0.259818 & -1.44948 \\
\hline & 0.750 & -0.273050 & 0.237089 & -1.15168 \\
\hline & 0.950 & -0.609036 & 0.285015 & -2.13686 \\
\hline \multirow[t]{5}{*}{ ORDEN } & 0.050 & 0.166667 & 0.260164 & 0.640621 \\
\hline & 0.250 & -0.0934783 & 0.182692 & -0.511670 \\
\hline & 0.500 & -0.130159 & 0.0941855 & -1.38194 \\
\hline & 0.750 & 0.0292553 & 0.0893321 & 0.327489 \\
\hline & 0.950 & -0.114985 & 0.163217 & -0.704490 \\
\hline
\end{tabular}

Cuadernos de Turismo, 41, (2018), 41-74 
En la tabla 8 y gráfico 1 observamos la posición de las pendientes de NOTA en cada una de las regresiones cuantiles para la cohorte 1.En la primera cohorte son significativas las pendientes de NOTA para los percentiles 50 (mediana) y 75 de ECO_I. En el primero, la pendiente es 0'18 mientras que para el percentil 75 dicha pendiente casi 0'30, lo que nos informa que en los percentiles superiores de las calificaciones de ECO_I el efecto de la NOTA es mayor.

\section{Gráfico 1 \\ POSICIÓN DE LAS PENDIENTES DE NOTA EN CADA UNA DE LAS REGRESIONES CUANTILES PARA LA COHORTE 1-ECO_I}

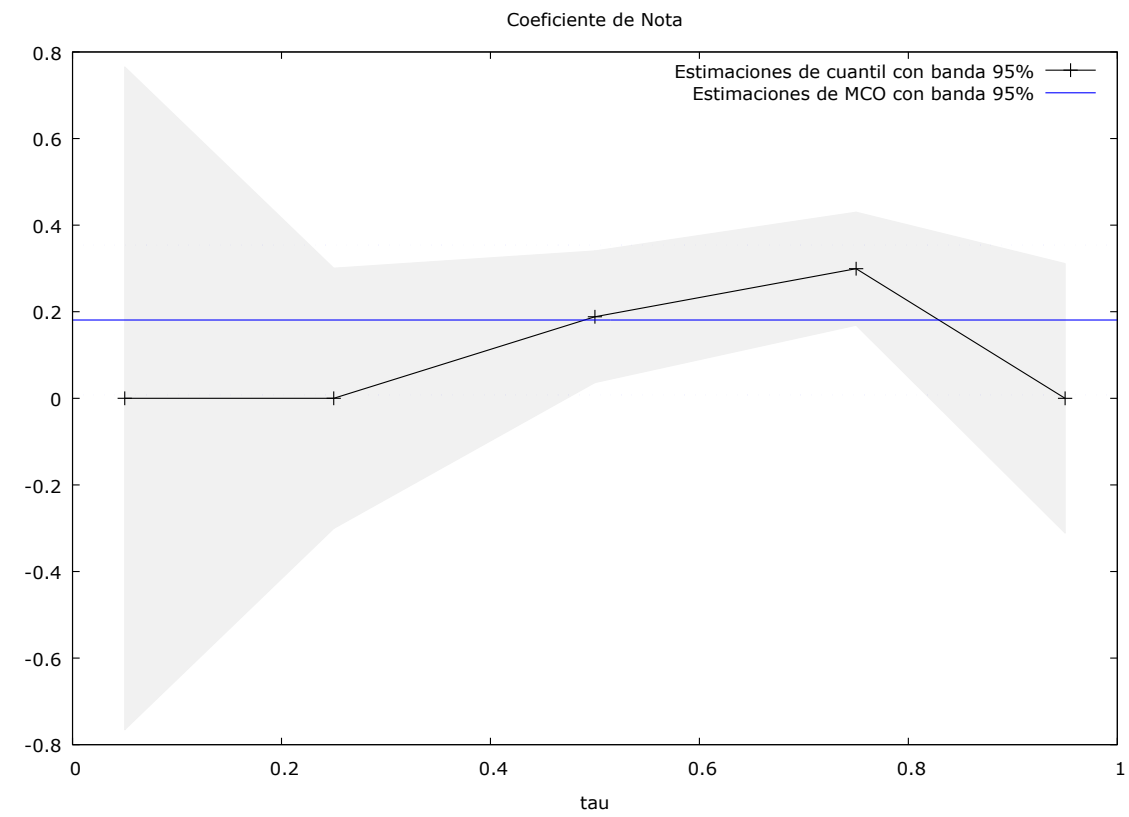

Por su parte, en la segunda cohorte (véase tabla 8 y gráfico 2) son significativas las pendientes de NOTA en todos los percentiles de ECO_I. Se observa que es importante la pendiente del percentil más bajo, que corresponde al 5\%, siendo significativa y con un valor 0'75. Recordemos que el cambio de una cohorte a otra se traduce en un incremento en la nota mínima de acceso. En el percentil 50 la pendiente es ahora 0’28, mientras que la misma en la cohorte 1 era 0'18, y en el percentil 75 dicha pendiente es 0'35 en esta cohorte número 2 ; mientras que para la cohorte 1 dicha pendiente es casi 0’30. Se sigue manteniendo la relación de que el efecto de NOTA es mayor en los percentiles superiores de ECO_I y, además, el hecho de que la nota mínima de acceso haya aumentado de 5'10 a 5'42 ha producido el incremento del efecto de la variable NOTA sobre los diferentes percentiles de la distribución de las calificaciones en la asignatura ECO_I. 


\section{Gráfico 2}

\section{POSICIÓN DE LAS PENDIENTES DE NOTA EN CADA UNA DE LAS REGRESIONES CUANTILES PARA LA COHORTE 2, ECO_I}

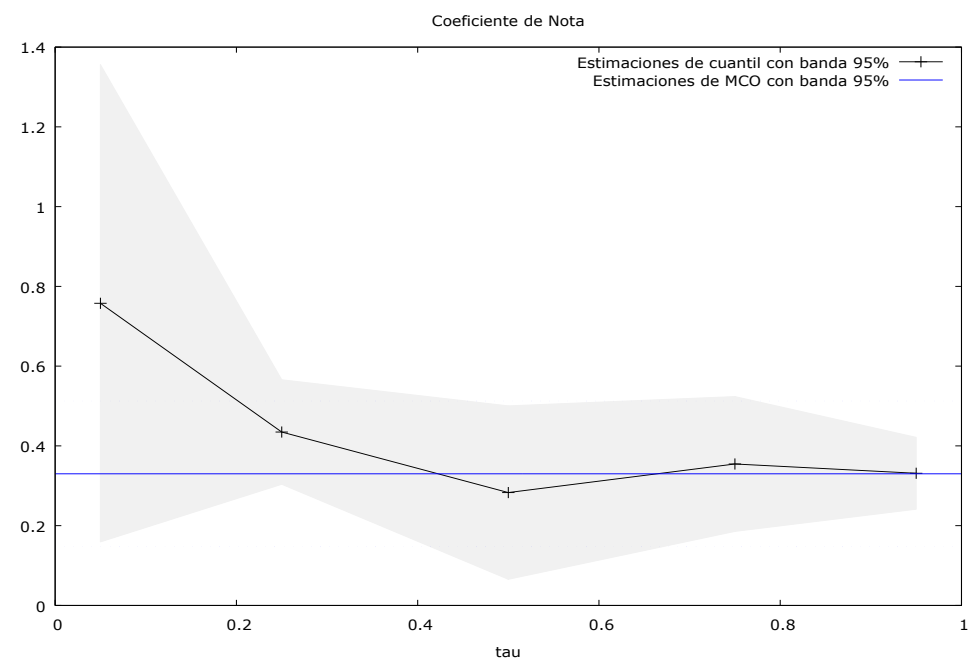

Tabla 9

ANÁLISIS DE REGRESIÓN MCO, ETMA

\begin{tabular}{llllll}
\hline & Coeficiente & Desv. Típica & \multicolumn{1}{c}{ Estadístico t } & \multicolumn{1}{c}{ Valor p } & \\
\hline Modelo 5. Observaciones 1-295. Primera cohorte .Variable dependiente: ETMA & \\
\hline const & 6.0442 & 0.550205 & 10.9854 & $<0.0001$ & $* * *$ \\
NOTA & 0.155376 & 0.0704962 & 2.2040 & 0.0284 & $* *$ \\
SEXO & 0.0371531 & 0.190902 & 0.1946 & 0.8458 & \\
ORDEN & -0.118744 & 0.0684996 & -1.7335 & 0.0841 & $*$ \\
\hline Modelo 6. Observaciones 1-277. Segunda cohorte .Variable dependiente: ETMA & \\
\hline const & 4.79015 & 0.475186 & 10.0806 & $<0.0001$ & $* * *$ \\
NOTA & 0.314394 & 0.058314 & 5.3914 & $<0.0001$ & $* * *$ \\
SEXO & -0.0107638 & 0.158033 & -0.0681 & 0.9457 & \\
ORDEN & -0.0867495 & 0.0578807 & -1.4988 & 0.1350 & \\
\hline
\end{tabular}

Para la variable ETMA, en la primera cohorte (tabla 9) son significativas las variables NOTA de acceso a la universidad (con una relación directa) y la variable ORDEN. Esta última con un valor $p=0.0841$, y con pendiente negativa, lo que nos informa del efecto contrario del número de orden en la petición del alumno sobre las calificaciones en esta asignatura. En la segunda cohorte la variable NOTA sigue siendo significativa con una pendiente que casi duplica a la de la primera (0’31 frente a 0'155), pero la variable ORDEN ya no lo es, aunque presenta de nuevo pendiente negativa. 
Tabla 10

ANÁLISIS DE REGRESIÓN CUANTIL, ETMA

\begin{tabular}{|c|c|c|c|c|}
\hline & tau & Coeficiente & Desv. Típica & Estadístico $t$ \\
\hline \multicolumn{5}{|c|}{ Modelo 7. Observaciones 1-295. Primera cohorte Variable dependiente: ETMA } \\
\hline \multirow[t]{5}{*}{ const } & 0.050 & 4.95000 & 2.70468 & 1.83016 \\
\hline & 0.250 & 5.88468 & 0.821438 & 7.16388 \\
\hline & 0.500 & 6.32798 & 0.474066 & 13.3483 \\
\hline & 0.750 & 6.94342 & 0.681846 & 10.1833 \\
\hline & 0.950 & 7.66928 & 0.905308 & 8.47146 \\
\hline \multirow[t]{5}{*}{ NOTA } & 0.050 & $3.20207 \mathrm{e}-012$ & 0.338682 & $9.45451 \mathrm{e}-012$ \\
\hline & 0.250 & 0.124611 & 0.0960686 & 1.29710 \\
\hline & 0.500 & 0.109037 & 0.0630760 & 1.72866 \\
\hline & 0.750 & 0.131579 & 0.0900287 & 1.46152 \\
\hline & 0.950 & 0.211864 & 0.0937560 & 2.25974 \\
\hline \multirow[t]{5}{*}{ SEXO } & 0.050 & $2.36325 \mathrm{e}-009$ & 1.01881 & $2.31961 \mathrm{e}-009$ \\
\hline & 0.250 & -0.106231 & 0.309180 & -0.343587 \\
\hline & 0.500 & 0.0148376 & 0.152931 & 0.0970221 \\
\hline & 0.750 & -0.184211 & 0.222765 & -0.826927 \\
\hline & 0.950 & -0.138771 & 0.388037 & -0.357624 \\
\hline \multirow[t]{5}{*}{ ORDEN } & 0.050 & -0.500000 & 0.401042 & -1.24675 \\
\hline & 0.250 & -0.239356 & 0.143797 & -1.66455 \\
\hline & 0.500 & -0.0409280 & 0.0505898 & -0.809017 \\
\hline & 0.750 & -0.0144737 & 0.0980806 & -0.147569 \\
\hline & 0.950 & -0.0497881 & 0.126589 & -0.393305 \\
\hline \multicolumn{5}{|c|}{ Modelo 8. Observaciones 1-277. Segunda cohorte Variable dependiente: ETMA } \\
\hline \multirow[t]{5}{*}{ const } & 0.050 & -0.399351 & 2.65264 & -0.150549 \\
\hline & 0.250 & 4.45488 & 0.459774 & 9.68928 \\
\hline & 0.500 & 5.74737 & 0.439275 & 13.0838 \\
\hline & 0.750 & 5.86065 & 0.512707 & 11.4308 \\
\hline & 0.950 & 5.58371 & 1.28459 & 4.34669 \\
\hline \multirow[t]{5}{*}{ NOTA } & 0.050 & 0.695733 & 0.278471 & 2.49841 \\
\hline & 0.250 & 0.295054 & 0.0466773 & 6.32115 \\
\hline & 0.500 & 0.199881 & 0.0507694 & 3.93703 \\
\hline & 0.750 & 0.270270 & 0.0677170 & 3.99117 \\
\hline & 0.950 & 0.455581 & 0.134927 & 3.37650 \\
\hline \multirow[t]{5}{*}{ SEXO } & 0.050 & 0.623840 & 0.898457 & 0.694347 \\
\hline & 0.250 & 0.0976022 & 0.139753 & 0.698389 \\
\hline & 0.500 & -0.173297 & 0.137809 & -1.25751 \\
\hline & 0.750 & -0.179003 & 0.148915 & -1.20205 \\
\hline & 0.950 & -0.505695 & 0.381420 & -1.32582 \\
\hline \multirow[t]{5}{*}{ ORDEN } & 0.050 & -0.0718924 & 0.390036 & -0.184322 \\
\hline & 0.250 & -0.125119 & 0.0545554 & -2.29342 \\
\hline & 0.500 & -0.0819762 & 0.0624855 & -1.31192 \\
\hline & 0.750 & -0.0579519 & 0.0508330 & -1.14005 \\
\hline & 0.950 & -0.0256264 & 0.121962 & -0.210118 \\
\hline
\end{tabular}




\section{Gráfico 3}

POSICIÓN DE LAS PENDIENTES DE NOTA EN CADA UNA DE LAS REGRESIONES CUANTILES PARA LA COHORTE 1, ETMA

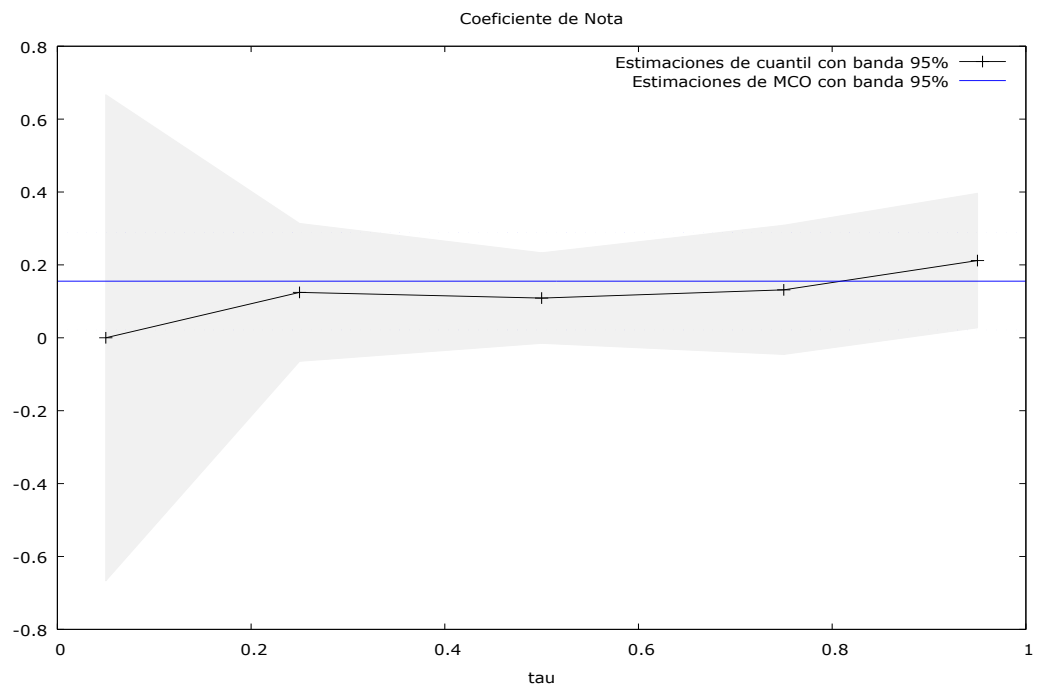

Gráfico 4

POSICIÓN DE LAS PENDIENTES DE NOTA EN CADA UNA DE LAS REGRESIONES CUANTILES PARA LA COHORTE 2, ETMA

Coeficiente de Nota

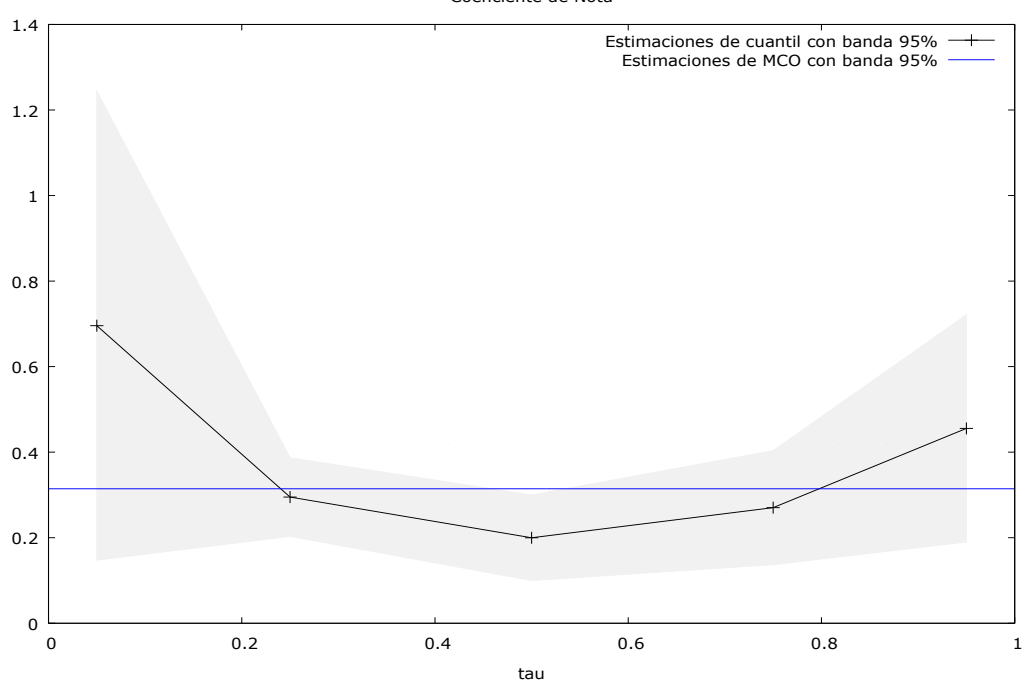

Cuadernos de Turismo, 41, (2018), 41-74 
En la tabla 10 se aprecia que, en cuanto a las regresiones cuantiles, en la primera cohorte solo es significativa la pendiente de NOTA en el percentil 95 de las calificaciones de ETMA. Dicha pendiente toma el valor 0’2118. En cambio, en la segunda cohorte son significativas las pendientes de NOTA en los cinco percentiles modelizados de estas calificaciones. En el caso particular del percentil 95 dicha pendiente toma el valor 0’45, más que duplicando el correspondiente valor de la primera cohorte. La pendiente del percentil 5 es la más elevada para la variable NOTA en la segunda cohorte. Al comparar las pendientes de ese percentil en ambas cohortes observamos el afecto del aumento de la nota de corte: sobre las calificaciones más bajas de esta asignatura tiene más peso la nota de acceso cuando la nota de corte es mayor. En ambas cohortes todas las pendientes de ORDEN, aunque no significativas, si son negativas: efecto contrario del orden de elección de los estudios de Grado en Turismo sobre las calificaciones de esta asignatura.

Tabla 11

ANÁLISIS DE REGRESIÓN MCO, EYOT

\begin{tabular}{lccccc}
\hline & Coeficiente & Desv. Típica & Estadístico t & Valor p & \\
\hline Modelo 9. Observaciones 1-295. Primera cohorte .Variable dependiente: EyOT & \\
\hline const & 2.31515 & 0.641816 & 3.6072 & 0.0004 & $* * *$ \\
NOTA & 0.410363 & 0.0787625 & 5.2101 & $<0.0001$ & $* * *$ \\
SEXO & 0.129691 & 0.213449 & 0.6076 & 0.5439 & \\
ORDEN & -0.0233211 & 0.0781772 & -0.2983 & 0.7657 & \\
\hline Modelo 10. Observaciones 1-277. Segunda cohorte .Variable dependiente: EyOT & \\
\hline const & 3.57431 & 0.573511 & 6.2323 & $<0.0001$ & $* * *$ \\
NOTA & 0.439668 & 0.0734823 & 5.9833 & $<0.0001$ & $* * *$ \\
SEXO & 0.353611 & 0.258989 & 1.3653 & 0.1567 & \\
ORDEN & -0.146824 & 0.0714011 & -2.0563 & 0.0407 & $* *$ \\
\hline
\end{tabular}

En la tabla 11 advertimos que en ambas cohortes es significativa la pendiente de NOTA, tomando el valor 0'41 en la primera y 0'44 en la segunda. La variable ORDEN es significativa en la segunda cohorte, y con pendiente negativa, o sea, al igual que se señaló antes, con efecto contrario sobre las calificaciones de EyOT.

Salvo la pendiente del percentil 5, el resto de pendientes de la variable NOTA son significativas como se observa en la tabla 12. Las más elevadas son las de los dos percentiles más altos, 0’49 y 0’48, por encima de la pendiente de la media estimada por MCO, 0`44. En este caso, entonces, la variable NOTA tiene más influencia sobre las calificaciones más altas de esta asignatura. El gráfico 5 nos muestra ambas pendientes por encima de la pendiente de la media, al igual que ocurre con las del percentil 25. 
Tabla 12

ANÁLISIS DE REGRESIÓN CUANTIL, EYOT

\begin{tabular}{|c|c|c|c|c|}
\hline & tau & Coeficiente & Desv. Típica & Estadístico t \\
\hline \multicolumn{5}{|c|}{ Modelo 11. Observaciones 1-295. Primera cohorte Variable dependiente: EyOT } \\
\hline \multirow[t]{5}{*}{ const } & 0.050 & 0.473096 & 1.42499 & 0.331999 \\
\hline & 0.250 & 1.03474 & 1.24395 & 0.831819 \\
\hline & 0.500 & 3.44996 & 0.71693 & 4.81210 \\
\hline & 0.750 & 2.64026 & 0.783926 & 3.36800 \\
\hline & 0.950 & 4.29757 & 1.00007 & 4.29725 \\
\hline \multirow[t]{5}{*}{ NOTA } & 0.050 & 0.279188 & 0.195476 & 1.42825 \\
\hline & 0.250 & 0.463516 & 0.164226 & 2.82243 \\
\hline & 0.500 & 0.299835 & 0.088199 & 3.39950 \\
\hline & 0.750 & 0.496748 & 0.098454 & 5.04546 \\
\hline & 0.950 & 0.485437 & 0.112867 & 4.30098 \\
\hline \multirow[t]{5}{*}{ SEXO } & 0.050 & -0.148731 & 0.438622 & -0.339087 \\
\hline & 0.250 & 0.581551 & 0.404335 & 1.43829 \\
\hline & 0.500 & -0.0335123 & 0.219426 & -0.152728 \\
\hline & 0.750 & -0.0640441 & 0.259074 & -0.247204 \\
\hline & 0.950 & 0.618447 & 0.367343 & 1.68357 \\
\hline \multirow[t]{5}{*}{ ORDEN } & 0.050 & 0.00101523 & 0.090841 & 0.0111758 \\
\hline & 0.250 & -0.249654 & 0.117833 & -2.11871 \\
\hline & 0.500 & -0.0467180 & 0.094355 & -0.495127 \\
\hline & 0.750 & 0.0637505 & 0.104835 & 0.608103 \\
\hline & 0.950 & 0.0451456 & 0.160736 & 0.280868 \\
\hline \multicolumn{5}{|c|}{ Modelo 12. Observaciones 1-277. Segunda cohorte Variable dependiente: EyOT } \\
\hline \multirow[t]{5}{*}{ const } & 0.050 & 1.49489 & 2.31013 & 0.647100 \\
\hline & 0.250 & 2.71745 & 0.943684 & 2.87962 \\
\hline & 0.500 & 4.40757 & 0.637906 & 6.90944 \\
\hline & 0.750 & 4.76924 & 0.639792 & 7.45436 \\
\hline & 0.950 & 6.58387 & 0.755113 & 8.71905 \\
\hline \multirow[t]{5}{*}{ NOTA } & 0.050 & 0.284091 & 0.308212 & 0.921740 \\
\hline & 0.250 & 0.436312 & 0.106629 & 4.09186 \\
\hline & 0.500 & 0.350631 & 0.0846848 & 4.14043 \\
\hline & 0.750 & 0.452675 & 0.0828232 & 5.46556 \\
\hline & 0.950 & 0.322581 & 0.0914026 & 3.52923 \\
\hline \multirow[t]{5}{*}{ SEXO } & 0.050 & 0.719318 & 0.951682 & 0.755838 \\
\hline & 0.250 & 0.447080 & 0.338072 & 1.32244 \\
\hline & 0.500 & 0.260589 & 0.218947 & 1.19019 \\
\hline & 0.750 & 0.210700 & 0.195129 & 1.07980 \\
\hline & 0.950 & 0.654839 & 0.380519 & 1.72091 \\
\hline \multirow[t]{5}{*}{ ORDEN } & 0.050 & -0.0306818 & 0.256288 & -0.119716 \\
\hline & 0.250 & -0.113160 & 0.129815 & -0.871700 \\
\hline & 0.500 & -0.154839 & 0.069256 & -2.23573 \\
\hline & 0.750 & -0.254424 & 0.055802 & -4.55940 \\
\hline & 0.950 & -0.0612903 & 0.135654 & -0.451814 \\
\hline
\end{tabular}

Cuadernos de Turismo, 41, (2018), 41-74 
Como viene ocurriendo hasta ahora en todas las estimaciones efectuadas, el sexo no es un factor relevante en estas calificaciones.

\section{Gráfico 5}

\section{POSICIÓN DE LAS PENDIENTES DE NOTA EN CADA UNA DE LAS} REGRESIONES CUANTILES PARA LA COHORTE 1, EYOT

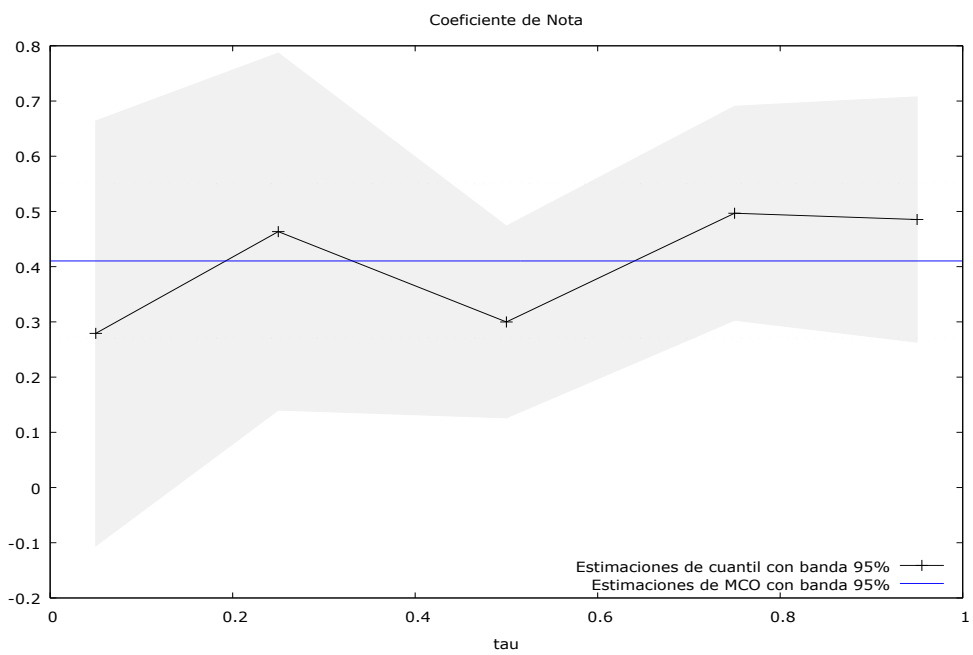

Gráfico 6

POSICIÓN DE LAS PENDIENTES DE NOTA EN CADA UNA DE LAS REGRESIONES CUANTILES PARA LA COHORTE 2, EYOT

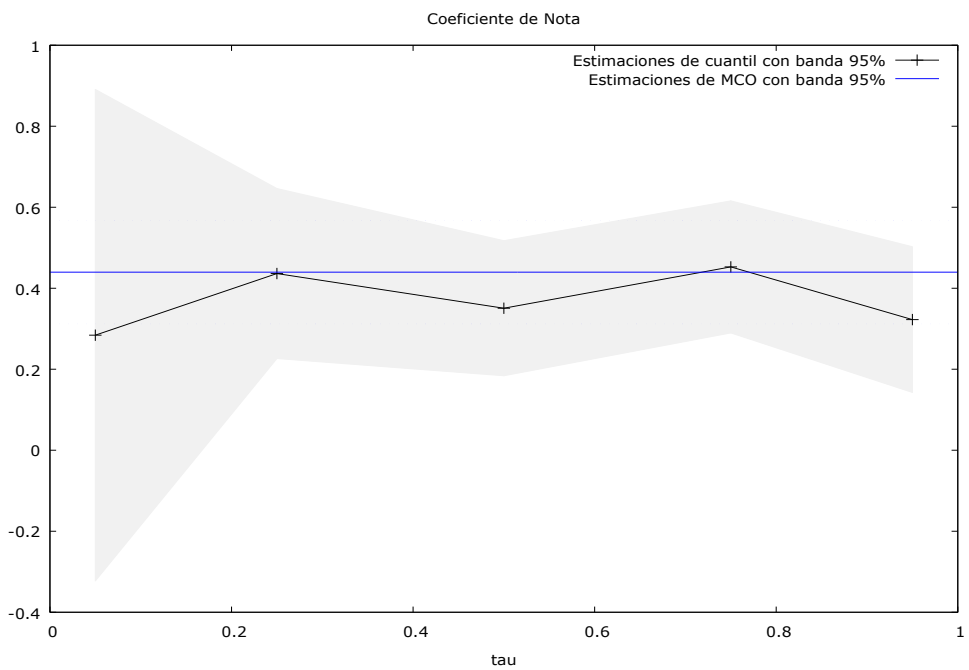


Al igual que ocurría en la primera cohorte, en esta segunda también son significativas las pendientes de los percentiles 25, 50, 75 y 95 de la variable NOTA. Asimismo, aparece como significativa, y con signo negativo, la pendiente del percentil 75 de la variable ORDEN.

Tabla 13

ANÁLISIS DE REGRESIÓN MCO, FDC

\begin{tabular}{llllll}
\hline & Coeficiente & Desv. Típica & \multicolumn{1}{c}{ Estadístico t } & Valor $\mathbf{p}$ & \\
\hline Modelo 13. Observaciones & 1-295. Primera cohorte & .Variable dependiente: $\mathbf{F d C}$ & \\
\hline const & 3.67982 & 0.567538 & 6.4838 & $<0.0001$ & $* * *$ \\
NOTA & 0.06185 & 0.0696473 & 0.8881 & 0.3752 & \\
SEXO & -0.17509 & 0.188747 & -0.9277 & 0.3544 & \\
ORDEN & 0.10681 & 0.0691297 & 1.5452 & 0.1234 & \\
\hline Modelo 14. & Observaciones 1-277. Segunda cohorte .Variable dependiente: FdC & \\
\hline const & 2.48447 & 0.599105 & 4.1470 & $<0.0001$ & $* * *$ \\
NOTA & $\mathbf{0 . 3 3 9 9 7}$ & $\mathbf{0 . 0 7 6 7 6 1 6}$ & $\mathbf{4 . 4 2 8 9}$ & $<\mathbf{0 . 0 0 0 1}$ & $* * *$ \\
SEXO & -0.01737 & 0.207869 & -0.0836 & 0.9335 & \\
ORDEN & -0.07630 & 0.0745875 & -1.0231 & 0.3072 & \\
\hline
\end{tabular}

Para la variable FdC, en la primera cohorte de la tabla 13, ninguna de las variables propuestas como explicativas resulta significativa en la modelización de las calificaciones de Fundamentos de Contabilidad de ese curso. En cambio, en la segunda sí vuelve a aparecer como significativa la variable NOTA, con una pendiente de 0’34.

Tabla 14

ANÁLISIS DE REGRESIÓN CUANTIL, FDC

\begin{tabular}{lcccc}
\hline & tau & Coeficiente & Desv. Típica & Estadístico t \\
\hline Modelo 15. Observaciones 1-295. Primera cohorte Variable dependiente: & FdC \\
\hline const & 0.050 & 0.58051 & 1.48066 & 0.39206 \\
& 0.250 & 3.44366 & 0.80940 & 4.25456 \\
& 0.500 & 4.09962 & 0.13331 & 30.7524 \\
& 0.750 & 4.05487 & 0.46110 & 8.79389 \\
\multirow{2}{*}{ NOTA } & 0.950 & 6.59585 & 2.61376 & 2.52350 \\
& 0.050 & 0.1298701 & 0.169280 & 0.767190 \\
& 0.250 & 0.0630888 & 0.087423 & 0.721648 \\
& 0.500 & 0.0123591 & 0.016181 & 0.763776 \\
SEXO & 0.750 & 0.0240708 & 0.049242 & 0.488824 \\
& 0.950 & 0.0638978 & 0.339843 & 0.188021 \\
& 0.050 & -0.301299 & 0.646552 & -0.466009 \\
& 0.250 & 0.128473 & 0.290711 & 0.441925
\end{tabular}




\begin{tabular}{lllll} 
& 0.500 & 0.004511 & 0.042613 & 0.105861 \\
ORDEN & 0.750 & 0.006980 & 0.171254 & 0.040761 \\
& 0.950 & -0.992971 & 0.838182 & -1.18467 \\
& 0.050 & 0.118182 & 0.144426 & 0.818287 \\
0.250 & 0.0221226 & 0.148757 & 0.148716 \\
& 0.500 & 0.0220921 & 0.0193558 & 1.14137 \\
& 0.750 & 0.156097 & 0.133718 & 1.16736 \\
\hline
\end{tabular}

\begin{tabular}{lllll}
\hline Modelo 16. Observaciones 1-277. Segunda cohorte Variable dependiente: & FdC \\
\hline const & 0.050 & 1.38168 & 0.910120 & 1.51813 \\
& 0.250 & 0.638348 & 0.892114 & 0.715546 \\
& 0.500 & 3.65258 & 0.750290 & 4.86822 \\
& 0.750 & 2.14676 & 0.750622 & 2.85998 \\
NOTA & 0.950 & 5.47317 & 1.25186 & 4.37202 \\
& 0.050 & 0.152672 & 0.148190 & 1.03024 \\
& $\mathbf{0 . 2 5 0}$ & $\mathbf{0 . 4 3 8 9 6 6}$ & $\mathbf{0 . 1 0 6 7 1 9}$ & $\mathbf{4 . 1 1 3 2 7}$ \\
& 0.500 & 0.183486 & 0.101177 & 1.81351 \\
SEXO & $\mathbf{0 . 7 5 0}$ & $\mathbf{0 . 5 0 9 7 0 9}$ & $\mathbf{0 . 0 9 3 6 4 4}$ & $\mathbf{5 . 4 4 3 0 0}$ \\
& 0.950 & 0.223547 & 0.166298 & 1.34425 \\
& 0.050 & -0.401527 & 0.199632 & -2.01133 \\
& 0.250 & -0.125917 & 0.351296 & -0.358435 \\
& 0.500 & 0.0496432 & 0.254682 & 0.194922 \\
& 0.750 & 0.0652913 & 0.277540 & 0.235250 \\
& 0.950 & 0.622802 & 0.537171 & 1.15941 \\
& 0.050 & -0.0862595 & 0.069672 & -1.23806 \\
& 0.250 & -0.134982 & 0.109108 & -1.23714 \\
& 0.500 & -0.0269903 & 0.120896 & -0.223252 \\
& 0.750 & 0.0131877 & 0.106588 & 0.123726 \\
& 0.950 & 0.0777943 & 0.174944 & 0.444682 \\
\hline \multirow{3}{*}{ ORDEN } & & & &
\end{tabular}

Del mismo modo que ocurría con la pendiente estimada por MCO de la media de las calificaciones de esta asignatura condicionada a la variable NOTA, también aquí las pendientes de los percentiles de las calificaciones de Fundamentos de Contabilidad con respecto a NOTA tampoco son significativos. El gráfico 7 nos muestra la proximidad de dichas pendientes a la pendiente de la media y al valor nulo.

Las pendientes de los percentiles 25 y 75 de estas calificaciones condicionadas a la variable NOTA son significativas, con valores elevados de 0'44 y 0'50, respectivamente, que superan la pendiente de la media, 0’33. 


\section{Gráfico 7}

POSICIÓN DE LAS PENDIENTES DE NOTA EN CADA UNA DE LAS REGRESIONES CUANTILES PARA LA COHORTE 1, FDC

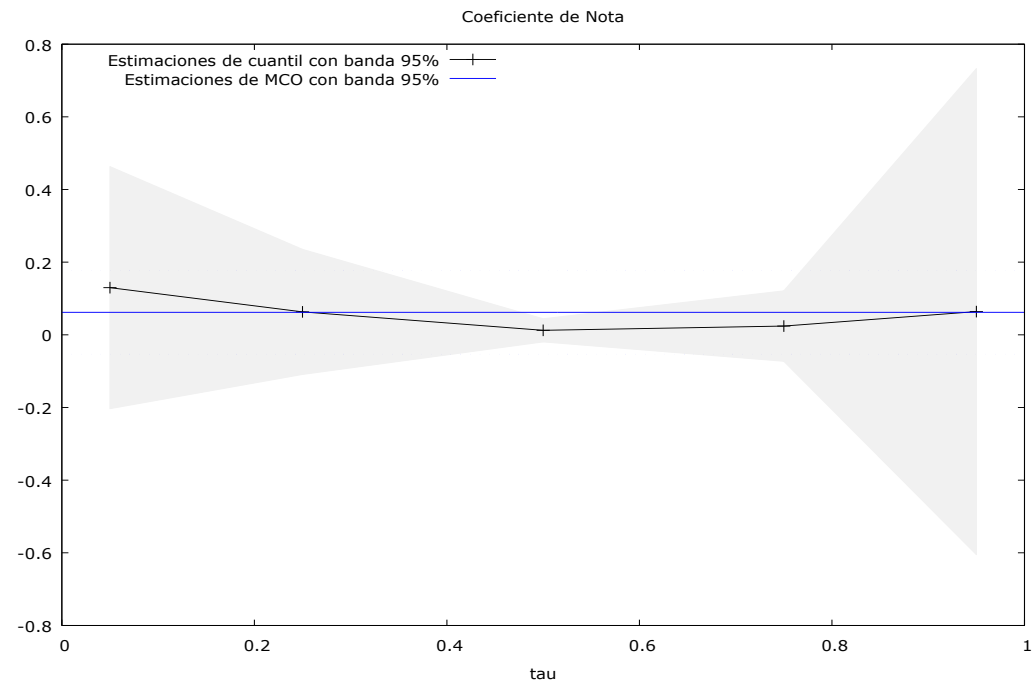

Gráfico 8

POSICIÓN DE LAS PENDIENTES DE NOTA EN CADA UNA DE LAS REGRESIONES CUANTILES PARA LA COHORTE 2, FDC

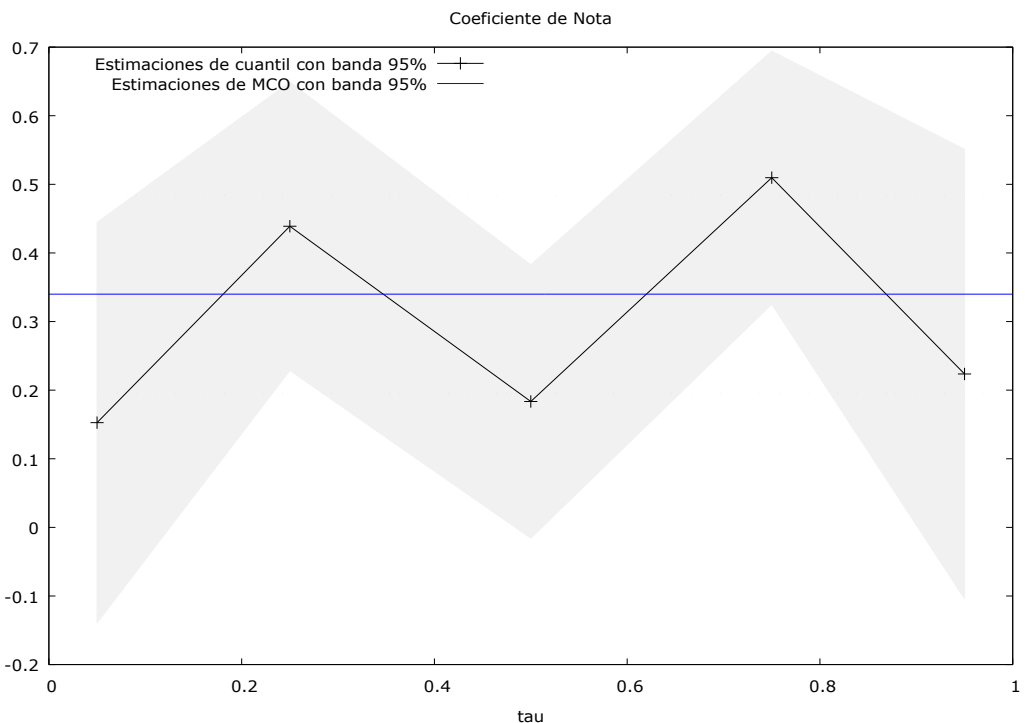

Cuadernos de Turismo, 41, (2018), 41-74 
Tabla 15

ANÁLISIS DE REGRESIÓN MCO, IAF

\begin{tabular}{lccccc}
\hline & Coeficiente & Desv. Típica & Estadístico t & Valor p & \\
\hline Modelo 17. Observaciones 1-295. Primera cohorte & .Variable dependiente: IaF & \\
\hline const & 2.71705 & 0.734484 & 3.6993 & 0.0003 & $* * *$ \\
NOTA & 0.300423 & 0.0901345 & 3.3331 & 0.0010 & $* * *$ \\
SEXO & -0.296123 & 0.244268 & -1.2123 & 0.2264 & \\
ORDEN & -0.102887 & 0.0894648 & -1.1500 & 0.2511 & \\
\hline Modelo 18. Observaciones 1-277. Segunda cohorte .Variable dependiente: IaF & \\
\hline const & 2.72054 & 0.761647 & 3.5719 & 0.0004 & $* * *$ \\
NOTA & 0.457183 & 0.0975877 & 4.6848 & $<0.0001$ & $* * *$ \\
SEXO & -0.119302 & 0.264265 & -0.4514 & 0.6520 & \\
ORDEN & -0.192691 & 0.0948238 & -2.0321 & 0.0431 & $* *$ \\
\hline
\end{tabular}

En la cohorte 1 de la tabla 15 la pendiente de las calificaciones medias de esta asignatura condicionadas a la variable NOTA es 0’30, mientras que en la cohorte 2 es 0’45, ambas significativas. Por tanto, en la segunda cohorte la variable NOTA de acceso tiene más influencia sobre las calificaciones de Introducción a las Finanzas. Además, en esta cohorte la variable ORDEN de elección es significativa y con pendiente negativa.

Tabla 16

ANÁLISIS DE REGRESIÓN CUANTIL, IAF

\begin{tabular}{lllll}
\hline & tau & Coeficiente & Desv. Típica & Estadístico t \\
\hline Modelo 19. Observaciones 1-295. Primera cohorte Variable dependiente: & IaF \\
\hline const & 0.050 & 2.38343 & 1.65120 & 1.44345 \\
& 0.250 & 4.72847 & 1.88454 & 2.50909 \\
& 0.500 & 4.49325 & 0.32018 & 14.0331 \\
& 0.750 & 1.91028 & 1.05504 & 1.81062 \\
\multirow{2}{*}{ NOTA } & 0.950 & 4.86991 & 1.54611 & 3.14978 \\
& 0.050 & -0.189873 & 0.217760 & -0.87193 \\
& 0.250 & -0.045558 & 0.230325 & -0.19779 \\
& 0.500 & 0.035023 & 0.049690 & 0.70483 \\
\multirow{2}{*}{ SEXO } & 0.750 & 0.548624 & 0.149287 & 3.67496 \\
& 0.950 & 0.470219 & 0.186006 & 2.52799 \\
& 0.050 & -0.211301 & 0.466373 & -0.453074 \\
& 0.250 & -0.948292 & 0.712741 & -1.33049 \\
& 0.500 & -0.0295911 & 0.043591 & -0.678821
\end{tabular}




\begin{tabular}{lllll} 
& 0.750 & -0.206576 & 0.324828 & -0.635953 \\
ORDEN & 0.950 & -0.0235110 & 0.486808 & -0.048296 \\
& 0.050 & 0.017744 & 0.164791 & 0.10767 \\
& 0.250 & -0.336219 & 0.236627 & -1.42088 \\
0.500 & -0.008101 & 0.011843 & -0.68406 \\
& 0.750 & -0.104991 & 0.067690 & -1.55105 \\
& 0.950 & -0.362069 & 0.190598 & -1.89965 \\
\hline
\end{tabular}

\begin{tabular}{lllll}
\hline Modelo 20. Observaciones 1-277. Segunda cohorte Variable dependiente: & IaF \\
\hline const & 0.050 & 2.37508 & 1.85403 & 1.28104 \\
& 0.250 & 0.64450 & 1.29279 & 0.49853 \\
& 0.500 & 2.60241 & 0.64347 & 4.04430 \\
NOTA & 0.750 & 3.27404 & 0.68458 & 4.78249 \\
& 0.950 & 8.57093 & 1.21579 & 7.04970 \\
& 0.050 & -0.061919 & 0.300914 & -0.20577 \\
& 0.250 & 0.595333 & 0.144018 & 4.13374 \\
SEXO & 0.500 & 0.482638 & 0.089041 & 5.42039 \\
& 0.750 & 0.540541 & 0.071163 & 7.59572 \\
& 0.950 & 0.116279 & 0.126748 & 0.91740 \\
& 0.050 & -0.402167 & 0.490728 & -0.81953 \\
& 0.250 & 0.508049 & 0.479073 & 1.06048 \\
ORDEN & 0.500 & 0.133874 & 0.186058 & 0.71953 \\
& 0.750 & -0.543017 & 0.250020 & -2.17189 \\
& 0.950 & -0.656977 & 0.386119 & -1.70149 \\
& 0.050 & -0.175851 & 0.179312 & -0.980701 \\
& 0.250 & -0.256929 & 0.309088 & -0.831247 \\
& 0.500 & -0.084961 & 0.062777 & -1.35337 \\
& 0.750 & -0.083502 & 0.090230 & -0.925438 \\
& 0.950 & -0.220930 & 0.104045 & -2.12342 \\
\hline
\end{tabular}

En el modelo 19 (tabla 16), las pendientes de los percentiles 75 y 95 de estas calificaciones, condicionadas a la variable NOTA, son significativas y con valores altos de 0'55 y 0'47, respectivamente, muy por encima de la pendiente estimada por MCO, que es de 0’30. En el gráfico 9 puede observarse este hecho, mientras que las otras tres quedan por debajo, no siendo significativas estas últimas.

En el modelo 20, se aprecia que las tres pendientes de los percentiles más centrales, 25, 50 y 75, de las calificaciones de esta asignatura condicionadas a NOTA son significativas. Además, esas tres pendientes se sitúan por encima de la estimada por MCO para la calificación media condicionada a NOTA, como se observa en el gráfico 10. 


\section{Gráfico 9}

POSICIÓN DE LAS PENDIENTES DE NOTA EN CADA UNA DE LAS REGRESIONES CUANTILES PARA LA COHORTE 1, IAF

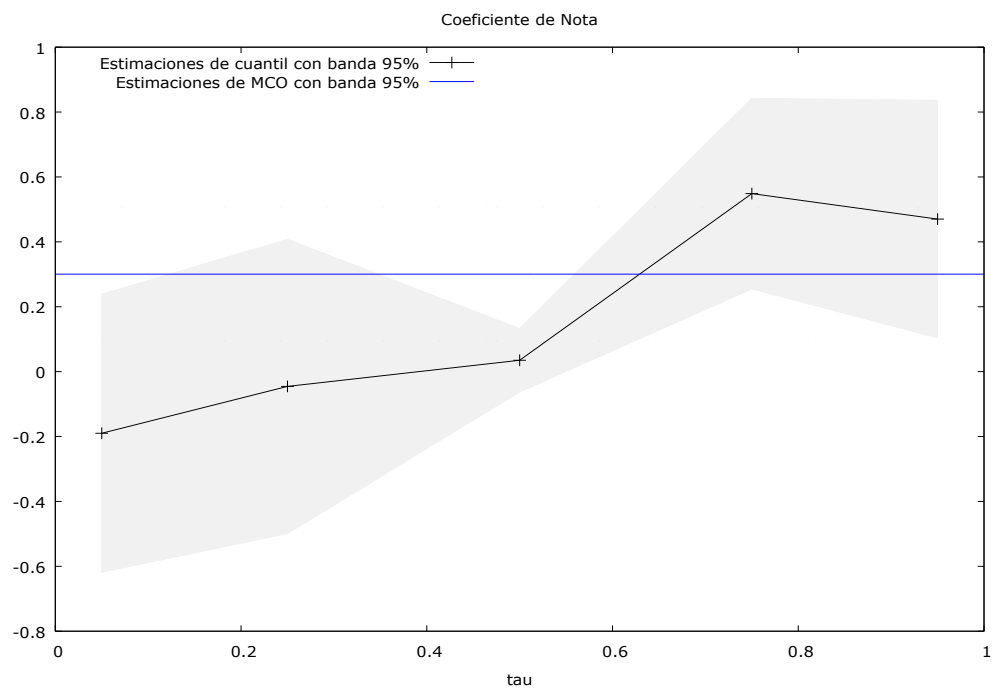

Gráfico 10

POSICIÓN DE LAS PENDIENTES DE NOTA EN CADA UNA DE LAS REGRESIONES CUANTILES PARA LA COHORTE 2, IAF

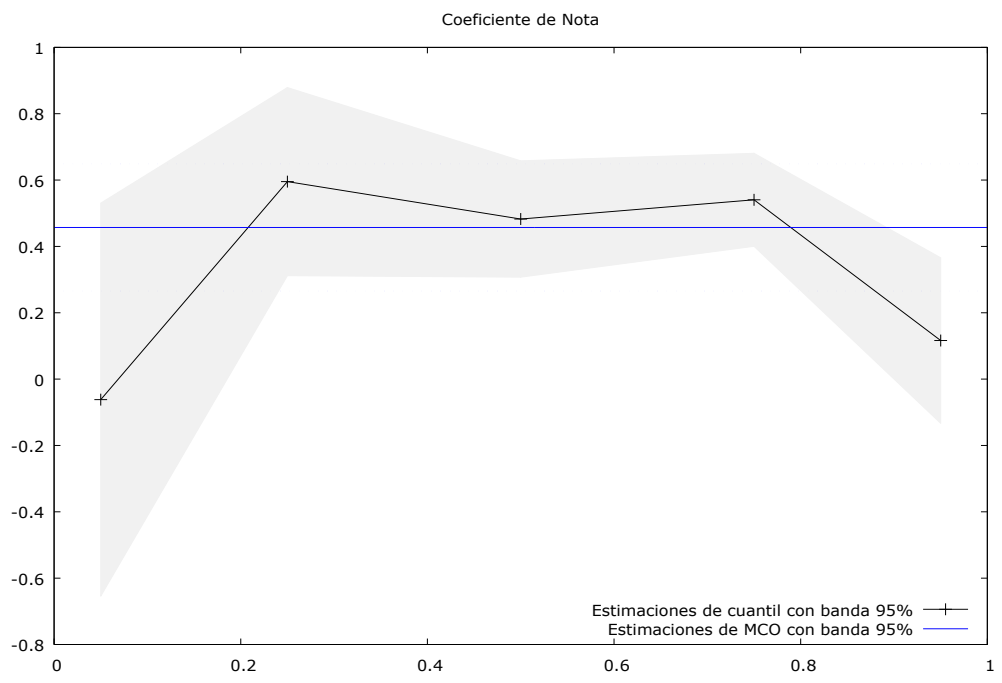




\section{CONCLUSIONES}

La regresión cuantil es un buen instrumento para modelizar valores distintos a la media de la variable objetivo en un análisis de regresión. Esos valores, al tratarse de cuantiles, están localizados. Es complementario a la estimación MCO clásica. Así es como lo hemos manejado en este trabajo.

Del análisis empírico de los datos podemos concluir lo siguiente, que coincide con los resultados de Jiménez-Caballero, Camúñez Ruiz, González-Rodríguez, \& Fuentes Ruiz, (2015) en el Grado en Finanzas y Contabilidad de la misma Facultad estudiada:

- El sexo no es una variable significativa que genere discriminación en las calificaciones de las asignaturas del primer cuatrimestre.

- Cuando el número de orden elegido en el Grado en Turismo -como opción de estudios universitarios- aparece como una variable significativa, la pendiente siempre ha sido negativa. Es decir, cuanto más alejada está la opción elegida de la primera posición, más bajas son las calificaciones de las asignaturas de ese primer cuatrimestre.

- La nota de acceso a la universidad es significativa y de pendiente positiva en casi todas las regresiones construidas. El paso de una cohorte a otra significaba un incremento en la nota mínima de acceso a los estudios de este grado de algo más de tres décimas. Pues bien, dicho aumento ha supuesto, en la mayoría de los casos, un aumento en la pendiente de NOTA en las regresiones de los diferentes cuantiles de las calificaciones de las cinco asignaturas analizadas. En algunos casos es destacable dicho aumento en los cuantiles más bajos de dichas calificaciones.

En resumen, los resultados obtenidos confirman la relevancia de dos factores como son la nota de acceso y orden de preferencia por la titulación, como predictores de las calificaciones obtenidas y, consecuentemente, de la satisfacción del estudiante con la carrera universitaria elegida, así como de mayor probabilidad de continuación en la misma. También, el aumento de la nota de corte hará que se condicione de manera aún más positiva las calificaciones en los tramos más bajos de las diferentes asignaturas con respecto a la nota de acceso. La escasez de estudios sobre rendimiento académico en carreras de turismo confiere a nuestro estudio utilidad en la capacidad de inferir el perfil más adecuado para los estudiantes de nuevo ingreso y con mayor probabilidad de éxito académico para el Grado en Turismo. La incorporación de estudiantes de nuevo ingreso con mayor motivación (orden de preferencia) y mejor nota de acceso permitiría afrontar el desafío de mejorar la formación académica, y realizar una mejor gestión de recursos públicos.

En referencia a la metodología, el modelo utilizado es más completo que el habitual de mínimos cuadrados ordinarios debido a que se estima la relación existente entre las variables explicativas y diferentes localizaciones de las variables objetivo, y no sólo la de la media. Hemos estimado por mínimos cuadrados ordinarios (minimizando errores al cuadrado) y minimizado errores absolutos ponderados. Los resultados, por tanto, son más ricos en información. 


\section{Implicaciones prácticas}

Los resultados parecen soportar argumentos que consideran que las instituciones universitarias deben adoptar medidas para la mejora del rendimiento académico. Establecer variables muy sensibles al mismo debe posibilitar un mejor ajuste entre la oferta y demanda académicas, y contribuir a formar mejores profesionales que encuentren mejores oportunidades de empleo en su sector.

\section{Implicaciones sociales}

Los resultados obtenidos sugieren que los recursos públicos, que son escasos, podrían asignarse de manera óptima y eficiente en el ámbito universitario del turismo. La sociedad debe percibir que los fondos dedicados a la formación universitaria de máxima cualificación se utilizan convenientemente. De esta forma, los recursos no asignados en este ámbito podrían dedicarse a potenciar ofertas formativas deficitarias, que profesional y socialmente ayudarían a mejorar el tejido empresarial e institucional.

\section{Limitaciones y futuras líneas de investigación}

El estudio tiene algunas limitaciones. En primer lugar, los datos corresponden a los observados en una Facultad concreta de turismo en España. Esto abre líneas de investigación para testar si ocurre algo similar en otras facultades. Además, se podrían añadir variables que mejoren el perfil de los estudiantes de turismo como puede ser conocer un idioma extranjero para estudiar en inglés o español (en Sevilla existe un itinerario en inglés), que, al mismo tiempo, contribuye a la empleabilidad.

\section{REFERENCIAS BIBLIOGRÁFICAS}

AITKEN, N.D. (1982): “College Student Performance, Satisfaction and Retention: Specification and Estimation of a Structural Model". The Journal of Higher Education, $53(1), 32-50$.

APODAKA, P. y GALLARETA, L.M. (1999): Propuesta de dos indicadores del acceso/ demanda de estudios universitarios en AA.VV.: Indicadores en la universidad: información y decisiones. Plan Nacional de Evaluación de la Calidad de las Universidades. Madrid: MEC-Consejo de Universidades.

BARTTOLUCCI, J. (1994): Desigualdad social, educación superior y sociología en México. México: CESU-UNAM/Porrúa.

BIRCH, E. R. y Miller, P.W. (2007): "A national study of students performance at university”. Australasian Journal of Economics Education, 4 (2), 223-236. https://doi. org/10.1108/09684881211240295

BUCHINSKY, M. (1995): Estimating the asymptotic covariance matrix for quantile regression models a Monte Carlo study. Journal of Econometrics, 68(2), 303-338. https://doi.org/10.1016/0304-4076(94)01652-G

CASCÓN, I.V. (1995): Análisis de las calificaciones escolares como criterio de rendimiento académico. Colegio Público Juan García Pérez.Gobierno de las Islas Canarias. http://www3.usal.es./inico/investigacion/jornadas/jornada2/comunc/cl7.html.

CHAÍN REVUELTAS, R. (1997): Ofertas, demandas y elecciones, en búsqueda de una profesión. In IV Congreso Nacional de Investigación Educativa (Ed.). Mérida: COMIE. 
CILLIERS, C.D. y Klerk, E. C. de. (2001): Theory-based prediction of academic performance at a South African university. International Journal of Special Education, 16(1).

COLEMAN, J.S., CAMPBELL, E.Q., HOBSON, C.J., MCPARTLAND, F., MOOD, A.M., y WEINFELD, F.D. (1966): Equality of Educacional Opportunity. Washington, D.C.: US Government Printing Office.

CORTADA DE KOHAN, N. (2005): El profesor y la orientación vocacional. México: Trillas.

DI GRESIA, L. (2007). Rendimiento académico universitario. Trabajo de tesis doctoral. Doctorado en Economía. Universidad Nacional de La Plata, Buenos Aires. Retrieved from http://digresia.com.ar/files/rendacadaaep/rendacadaaep_full.pdf

EDEL-NAVARRO, R. (2003): EL rendimiento académico: concepto, investigación y desarrollo. REICE - Revista Electrónica Iberoamericana Sobre Calidad, Eficacia Y Cambio En Educación, 1(2), 1-15. https://doi.org/2152

FRENDE VEGA, M.Á., BIEDMA FERRER, J.M. y ARANA JIMÉNEZ, M. (2017): Influencia de la percepción y metodologías docentes aplicadas en el rendimiento académico de los estudiantes de la asignatura de Dirección de Recursos Humanos en las Empresas Turísticas. Cuadernos de Turismo, 39, 149-166. Retrieved from http:// dx.doi.org/10.6018/turismo.39.290481

GARBANZO, G.M. (2007): Factores asociados al rendimiento académico en estudiantes universitarios, una reflexión desde la calidad de la educación superior pública. Educación, 31(1), 43-63. https://doi.org/10.15517/revedu.v31i1.1252

GARCÍA, M. M. y SEGUNDO, M. J. (2001): El rendimiento académico en el primer curso universitario. In X Jornadas de la Asociación de Economía de la Educación (Ed.) (pp. 435-446). Murcia. Retrieved from ftp://ftp.puce.edu.ec/Facultades/CienciasEducacion/ Maestria/CienciasEducacion/Economía de la Educación/Garcia_SanSegundo.pdf

HÄKKINEN, L. (2004): Do University Entrance Exams Predict Academic Achievement? Working Paper, Department of Economics, Uppsala University, No. 2004:16. Retrieved from https://www.econstor.eu/bitstream/10419/82773/1/wp2004-016.pdf

HASAN, M. N., \& KOENKER, R. . W. (1997): Robust Rank Tests of the Unit Root Hypothesis. Econometrica, 65(1), 133-161. Retrieved from http://www.jstor.org/stable/pdf/2171816.pdf?refreqid=excelsior\%3A3bc60708e805f8457ebbf7b898428e5c

HECKMAN, J. J. (1979): Sample Selection Bias as a Specification. Econometrica, 47(1), 153-161. Retrieved from http://0-www.jstor.org.fama.us.es/stable/pdf/1912352.pdf

HILL, R. C., GRIFFITHS, W. E., \& LIM, G. C. (2012): Principles of Econometrics (4th ed.). Chennay (India): John Wiley \& Sons. Retrieved from https://gettextbook.download/Econ 103/Principles of Econometrics - 4th Edition.pdf

HUBER, P. J. (1967): The behavior of maximum likelihood estimates under nonstandard conditions. In LeCam L.M. y Neyman J. (Ed.) (pp. 221-233). Berkeley, California: Fifth Berkeley Symposium on Mathematical Statistics y Probability, Vol. 1, University of California Press.

ISMAIL, N. A., \& OTHMAN, A. (2006): Comparing university academic performances of HSC students at the three art-based faculties. International Education Journal, 7(5), 668-675. Retrieved from http://files.eric.ed.gov/fulltext/EJ854324.pdf 
JIMÉNEZ-CABALLERO, J. L., CAMÚÑEZ RUIZ, J. A., GONZÁLEZ-RODRÍGUEZ, M. R., \& FUENTES RUIZ, P. (2015): Factores determinantes del rendimiento académico universitario en el Espacio Europeo de Educación Superior. Innovar: Revista de Ciencias Administrativas Y Sociales, 25(58), 159-175. https://doi.org/10.15446/ innovar.v25n58.52440.ENLACE

JIMÉNEZ, M. (1994): Competencia social: intervención preventiva en la escuela. Infancia $Y$ Sociedad:Revista de Estudios,24(October),21-48. Retrieved from https://www.researchgate. net/publication/259442484_Competencia_social_intervencion_preventiva_en_la_escuela

KOENKER, R. (2005): Quantile Regression. Cambridge Books, 15(4), 366. Retrieved from http://ideas.repec.org/b/cup/cbooks/9780521608275.html

KOENKER, R., \& Basset, G. (1978). Regression Quantiles. Econométrica, 46(1), 33-50. Retrieved from http://0-www.jstor.org.fama.us.es/stable/pdf/1913643.pdf

KOENKER, R., \& HALLOCK, K. F. (2001). Quantile Regression. Journal of Economics Perspectives, 15(4), 143-156. Retrieved from http://ideas.repec.org/b/cup/ cbooks/9780521608275.html

KOENKER, R., \& MACHADO, J. A. F. (1999). Goodness of fit and related inference processes for quantile regression. Journal of the American Statistical Association, 94(448), 1296-1310.

KOENKER, R., NG, P., \& PORTNOY, S. (1994). Quantile Smoothing Splines. Biometrika Trust, 81(4), 673-680.

KOENKER, R., \& ZHAO, Q. (1994). L-Estimation for linear heteroscedastic models. Journal of Nonparametric Statistics, 3(3-4), 223-235. https://doi. org/10.1080/10485259408832584

KWEK, A., BUI, H. T., RYNNE, J., \& SO, K. K. F. (2013). The impacts of self-esteem and resilience on academic performance: An investigation of domestic and international hospitality and tourism undergraduate students. Journal of Hospitality and Tourism Education, 25(3), 110-122. https://doi.org/10.1080/10963758.2013.826946

LUNAR LEANDRO, R. A., \& MARCANO LÓPEZ, V. R. (2007). Caracterización del perfil motivacional del estudiante de ingreso a la carrera de Turismo y Hotelería, Universidad de Oriente, Núcleo Nueva Esparta, Año 2001. Gestión Turística, 7, 6-26. https://doi.org/10.4206/gest.tur.2007.n7-01

MIGUEL DÍAZ, M. DE, APODACA, P., ARIAS, J. M., ESCUDERO, T., RODRÍGUEZ, S., \& VIDAL, J. (2002). Evaluación del Rendimiento en la Enseñanza Superior. Comparación de resultados entre alumnos procedentes de la Logse y del COU. Revista de Investigacion Educativa, 20(2), 357-383. Retrieved from https://www.researchgate. net/publication/41570267_Evaluacion_del_rendimiento_en_la_ensenanza_superior_ Comparacion_de_resultados_entre_alumnos_procedentes_de_la_LOGSE_y_del_COU MONTERO ROJAS, E., VILLALOBOS PALMA, J., \& VALVERDE BERMÚDEZ, A. (2007). Factores Institucionales, pedagógicos, psicosociales y soxciodemográficos asociados al rendimiento académico en la Universidad de Costa Rica: un análisis multinivel. Relieve, 13(2), 215-234. Retrieved from www.uv.es/RELIEVE/\%0Av13n2/ RELIEVEv13n2_5.htm

MOSTELLER, F., \& TUKEY, J. W. (1977). Data analysis and regression: a second course in statistics. Reading, MA: Addison-Wesley. 
OlADOKun, V. O., ADEBAnjO, A. T., \& CHARLES-OWABA, O. E. (2008). Predicting Students' Academic Performance using Artificial Neural Network: A Case Study of an Engineering Course. The Pacific Journal of Science and Technology, 9(1), 72-79. Retrieved from http://www.akamaiuniversity.us/PJST.htm\%0Ahttp://pdf.aminer. org/000/567/006/using_artificial_neural_nets_to_predict_academic_performance.pdf

PIÑEROS JIMÉNEZ, L. J., \& RODRÍGUEZ PINZÓN, A. (1998). Los insumos escolares en la educación secundaria y su efecto sobre el rendimiento académico de los estudiantes: un estudio en Colombia. The World Bank. Latin America and the Caribbean Regional Office Paper Series $n^{\circ} 36$. Washington D.C. Retrieved from http://www-wds. worldbank.org/external/default/WDSContentServer/WDSP/IB/2000/10/27/000094946 _0010130548125/Rendered/PDF/multi_page.pdf

POWELL, J. L. (1989). Estimation of monotonic regression models under quantile restrictions in Non-parametric and Semiparametric Methods in Econometrics. In W. Barnett, J. Powell, \& G. Tauchen (Eds.). Cambridge: Cambridge University Press.

RODRÍGUEZ, S., FITA, E., \& TORRADO, M. (2004). El Rendimiento Académico en la transición secundaria - Universidad. Revista de Educación, 6-10. Retrieved from http://www.revistaeducacion.educacion.es/re334/re334_22.pdf

SALANOVA SORIA, M., MARTÍNEZ MARTÍNEZ, I. M., BRESÓ ESTEVE, E., LLORENS GUMBAU, S., \& GRAU GUMBAU, R. (2005). Bienestar psicológico en estudiantes universitarios: Facilitadores y obstaculizadores del desempeño académico. Anales de Psicología, 21(1), 170-180.

STEPHEN, D. F., WELMAN, J. C., \& JORDAAN, W. . J. (2004). English language proficiency as an indicator of academic performance at a tertiary institution. Journal of Human Resource Management, 2(3), 42-53.

TINTO, V. (1995). Keynote address to the Inaugural Pan Pacific First Year Experience Conference. Brisbane (Australia).

TOCA, M. T., \& TOURÓN, J. (1989). Factores del rendimiento académico en los estudios de arquitectura. Revista de Investigación Educativa, 7(14), 31-47.

TOURÓN, J. (1984). Factores del rendimiento académico en la Universidad. Navarra: Ediciones Universidad de Navarra.

VALDERAS, J. M., OLMEDO, E., \& RODRÍGUEZ, M. J. (2009). Rendimiento académico comparado del programa de enseñanzas regladas en lengua inglesa en la EUEE de la Universidad de Sevilla. In J. L. Jiménez-Caballero \& M. Sacristán-Díaz (Eds.), Docencia en lengua inglesa en la Escuela de Estudios Empresariales de la Universidad de Sevilla: Implantación y resultados (pp. 89-111). Granada: Grupo Editorial Universitario.

VALLE, M. A., \& MARCIA, S. (1995). La demanda de carreras profesionales de los estudiantes de tercer año de bachillerato de ciudad Netzahualcóyotl, ciclo 1989-1990. México: CISE-UNAM.

VELO CONDE, C. A. (2004). La orientación vocacional y el proceso de elección de carrera. Tesis de licenciatura en Pedagogía, Universidad Pedagógica Nacional Unidad Ajusco, México.

WOOLRIDGE, J.M. (2010). Introducción a la econometría: Un enfoque moderno (4 edición). México: Cengage Learning Editores, S.A. de C.V. 